\title{
Roger Fry et Clive Bell : divergences fondamentales autour de la notion de «Significant Form "
}

\section{Pierrette Jutras}

Volume 20, numéro 1-2, 1993

URI : https://id.erudit.org/iderudit/1072762ar

DOI : https://doi.org/10.7202/1072762ar

\section{Aller au sommaire du numéro}

\section{Éditeur(s)}

UAAC-AAUC (University Art Association of Canada | Association d'art des universités du Canada)

\section{ISSN}

0315-9906 (imprimé)

1918-4778 (numérique)

\section{Découvrir la revue}

\section{Citer cet article}

Jutras, P. (1993). Roger Fry et Clive Bell : divergences fondamentales autour de la notion de «Significant Form ». RACAR : Revue d'art canadienne / Canadian Art Review, 20(1-2), 98-115. https://doi.org/10.7202/1072762ar

\section{Résumé de l'article}

The fundamental differences between the formalism of Roger Fry and that of Clive Bell far outweigh the few part of communalities that are often recalled to fuse, and confuse, one with the other. More specifically, the axioms which underwrite their respective views of "significance," "form," "significant and expressive form" or "Significant Form," imply orientations and meanings which are so antithetic that they must be read as opposing rather than supporting each other. Such is the global conclusion resulting from a comparative analysis of texts from both authors written over a period of some twenty-five years (1909-1934). This analysis was conducted around such themes as the relationship of art and life, the means and methods of access to the aesthetic experience, and the manifestations and finalities of those experiences. The findings are fourfold. First, while Fry wants art to serve as a "double life," unfaillingly rooted in and attentive to the human experience in this world, Bell feels art to be entirely devoted to "another world" and totally independent of - and oblivious to - the human experience. Second, while Fry presumes that anyone willing to work at learning and developing visual and perceptual skills can gain access to the aesthetic experience, Bell declares that only those born with the "aesthetic sensibility" will ever reach its peaks. Third, while Fry's aesthetic experiences produce a variety of affects of different intensities (feelings or emotions, Bell's "grand aesthetic thrill" is unique and always possessed of ecstatic intensity. Fourth, while Fry involves art in a process of cognition and communication, Bell glorifies art as a refuge and an escape from all communication and articulated cognition. Obviously, then, the significances and forms envisaged and supported within each of these contexts are irreconcilable.
Tous droits réservés (C) UAAC-AAUC (University Art Association of Canada | Association d'art des universités du Canada), 1994
Ce document est protégé par la loi sur le droit d'auteur. L'utilisation des services d’Érudit (y compris la reproduction) est assujettie à sa politique d'utilisation que vous pouvez consulter en ligne.

https://apropos.erudit.org/fr/usagers/politique-dutilisation/ 


\title{
Roger Fry et Clive Bell: divergences fondamentales autour de la notion de "Significant Form"*
}

\author{
Pierrette Jutras, Université du Québec a Montréal
}

\begin{abstract}
$\square$

he fundamental differences between the formalism of Roger Fry and that of Clive Bell far outweigh the few part of communalities that are often recalled to fuse, and confuse, one with the other. More specifically, the axioms which underwrite their respective views of "significance,"' 'form,' "'significant and expressive form" or "Significant Form," imply orientations and meanings which are so antithetic that they must be read as opposing rather than supporting each other. Such is the global conclusion resulting from a comparative analysis of texts from both authors written over a period of some twenty-five years (1909-1934). This analysis was conducted around such themes as the relationship of art and life, the means and methods of access to the aesthetic experience, and the manifestations and finalities of those experiences. The findings are fourfold. First, while Fry wants art to serve as a "double life," unfaillingly rooted in and
\end{abstract}

attentive to the human experience in this world, Bell feels art to be entirely devoted to "another world" and totally independent of--and oblivious to - the human experience. Second, while Fry presumes that anyone willing to work at learning and developing visual and perceptual skills can gain access to the aesthetic experience, Bell declares that only those born with the "aesthetic sensibility" will ever reach its peaks. Third, while Fry's aesthetic experiences produce a variety of affects of different intensities (feelings or emotions, Bell's "grand aesthetic thrill" is unique and always possessed of ecstatic intensity. Fourth, while Fry involves art in a process of cognition and communication, Bell glorifies art as a refuge and an escape from all communication and articulated cognition. Obviously, then, the significances and forms envisaged and supported within each of these contexts are irreconcilable. 'idée d'une signifiance de la forme visuelle, portée par l'expression significant and expressive form ${ }^{i}$ ou par celle de Significant Form ${ }^{2}$, renvoie aux débuts du formalisme anglais et associe les deux critiques d'art britanniques, Roger Fry (1866-1934) et Clive Bell (1881-1964). Malgré une alliance apparente, les rapports entre les pensées de ces deux auteurs restent ambigus. Même si la plupart des commentateurs s'accordent pour reconnaître des différences, certains rapprochements persistent en dépit du fait qu'ils ne se fondent que sur des fragments, peu représentatifs à notre avis, de la pensée globale des deux auteurs ${ }^{3}$. Cet article tentera donc de dissoudre ces ambiguïtés et d'établir que les propositions esthétiques de Fry et de Bell s'avèrent nettement divergentes.

Afin de bien distinguer les tendances constantes des hésitations ou des contradictions passagères, nous avons étudié des textes, produits sur une période d'environ vingtcinq ans ${ }^{4}$. Cette distinction importe puisque, d'emblée, nous considérons ces tendances - c'est-à-dire ces positions axiomatiques plus ou moins affichées - comme déterminantes en ce qui concerne les signifiances et les formes envisageables selon les points de vue de Fry ou de Bell. Ces positions axiomatiques ressortent le plus clairement à travers les thèmes récurrents du rapport art/vie, ou des conditions et moyens d'accès à l'expérience esthétique, ou encore des effets et des finalités reliés ce type d'expérience. Ces thèmes servent donc de structure au développement de notre analyse.

Certes, quelques éléments de convergence historique permettent une mise en tandem de Fry et de Bell. A un même moment (principalement entre 1910 et 1914), à un même endroit (en Angleterre), et initialement autour des mêmes événements artistiques et médiatiques (les premières expositions "post-impressionnistes"), Roger Fry et Clive Bell ont mené conjointement l'une des premières et des plus efficaces campagnes de promotion de l'art moderne et de la critique dite "formaliste". Ils défendent alors à peu près les mêmes artistes (les "post-impressionnistes" français principalement ${ }^{5}$ ), dénoncent les mêmes pratiques académiques et proclament la primauté de l'aspect formel sur le contenu narratif. De plus, le type de sens recherché dans l'oeuvre se voit spécifié par l'adjonction d'une autre composante, celle de l'émotion, que les deux auteurs posent comme un lieu de manifestation et de formation du sens. Puisque, par définition, l'émotion renvoie hors du raisonné et en marge d'un "vécu conscient" bien contrôlé et bien assimiléb, il ne s'agit donc pas de chercher dans l'oeuvre, à la manière de Panofsky, par exemple, l'élucidation d'une énigme d'ordre philologique et relevant d'un déjà-su verbal, susceptible d'être restauré par l'érudition. Au contraire, les émotions que Fry et que Bell soulignent, relèveraient plutôt d'un lieu où le verbal vacille, balbutie ou s'éteint. Cette affirmation d'un sens envahi par l'affectivité témoigne d'une autre offensive commune, contre l'hégémonie rationaliste et contre une définition du sens qui n'admettrait que ce qui s'établit de manière consciente. Outre ces quelques points d'entente, l'utilisation des mêmes mots, aussi vagues et ambigus que form, significant ou emotion ${ }^{7}$, accroît l'impression d'un accord global ${ }^{8}$ qui, en fait, n'existe pas. Aussitôt franchi le seuil des généralités historiques, puis celui d'une esthétique formaliste, les différences s'affirment et les auteurs 
bifurquent vers des conceptions formalistes incompatibles où la signifiance ainsi que la forme auxquelles ils se réferent sont de nature et de fonction diamétralement opposées. Dans la préface d'Art, Bell parle de désaccords profonds et durables avec Fry concernant les principes de l'esthéti$q^{2} e^{9}$. C'est l'analyse de ces divergences, ancrées dans le plan irréductible des axiomes, qui constitue l'essentiel de notre propos.

\section{RAPPORT ART-VIE (LIEU DE L'EXPÉRIENCE ESTHÉTIQUE)}

A propos des philosophies de l'art, énoncées en Allemagne au $19 \mathrm{e}$ et au début du 20e siècles, Michael Podro dégage deux tendances principales: une première - d'inspiration hégélienne - qui retire l'art de la vie active pour le réserver à des fins strictement personnelles et contemplatives, et une deuxième, tout à l'opposé, qui insiste plutôt pour intégrer l'art dans la vie active et l'utiliser à des fins sociales ${ }^{10}$. Bell, avec débordement dans $A r t$, puis avec une persistance plus ou moins affichée dans ses textes ultérieurs, adopte la voie hégélienne, celle de la transcendance, de l'absolu, de la finalité -dite-sans-fin- et de l'expérience spirituelle strictement personnelle. Fry, par contre, refuse ces deux tendances et choisit une troisième voie, plus en accord avec la tradition de l'empirisme britannique, qui privilégie l'approche scientifique et propose d'ouvrir sur la psychologie expérimentale et éventuellement, peut-être, sur la psychanalyse ${ }^{11}$. Cette tradition empiriste qui, depuis longtemps déjà, pose l'imagination comme une faculté constructive, offre à Fry un lieu médian, propice à son type d'expérience esthétique. Cette expérience esthétique, sans vouloir la soumettre directement à un principe d'utilité sociale, Fry en fait néanmoins un outil de communication inter-personnelle, à mi-chemin entre le privé et le public.

Dans "An Essay in Aesthetics" (1909), Fry indique l'étendue de l'expérience humaine qu'il inclut dans le champ de l'expérience esthétique lorsqu'il ajoute, après avoir présenté ce qu'il nomme les "emotional elements of design:

[...] it will be noticed that nearly all these emotional elements of design are connected with essential conditions of our physical existence: rhythm appeals to all the sensations which accompany muscular activity; mass to all the infinite adaptations to the force of gravity which we are forced to make; the spatial judgment is equally profound and universal in its application to life; our feeling about inclined planes is connected with our necessary judgments about the conformation of the earth itself; light, again, is so necessary a condition of our existence that we become intensely sensitive to changes in its intensity. Colour is the only one of our elements which is not of critical or universal importance to life, and its emotional effects is neither so deep nor so clearly determined as the others. It will be seen, then, that the graphic arts arouse emotions in us by playing upon what one may call the overtones of some of our primary physical needs. ${ }^{12}$

Fry entend donc relier l'esthétique au sensible et suivre ces liens jusqu'au niveau des phénomènes les plus primaires, c'est-à-dire jusqu'au niveau sensoriel (physique et physiologique). Quinze ans plus tard, dans "Pure and Impure Art" - un article fort ambigu où Fry semble d'abord tendre vers une séparation de l'art et de la vie - il finit par se raviser dans le tout dernier paragraphe et termine sa réflexion par un questionnement qui défait l'hypothèse puriste:

But in art there is, I think, an affective quality which lies
outside that. It is not a mere recognition of order and in-
terrelation; every part, as well as the whole, become suf-
fused with an emotional tone. Now, from our definition of
[... pure beauty, the emotional tone is not due to any rec-
ognizable reminiscence or suggestion of the emotional ex-
periences of life; but I sometimes wonder if it nevertheless
does not get its force from arousing some very deep, very
vague, and immensely generalized reminiscences. It looks
as though art had got access to the substratum of all the
emotional colors of life, to something which underlies all
the particular and specialized emotions of actual life. It
seems to derive an emotional energy from the very condi-
tions ofour existence by its relation of an emotional signif-
cance in time and space, or it may be that art really calls
up, as it were, the residual traces left on the spirit by the
different emotions of life, without however recalling the
actual experiences, so that we get an echo of the emotion
without the limitation and particular direction which it
had in experience. ${ }^{13}$

Depuis le texte de 1909, l'insistance sur le rapport entre le plastique et le physique s'est atténuée et Fry met maintenant l'accent sur des notions d'ordre psychologique (affective, reminiscences, [echo], substratum, residual traces). Ces notions soutiennent toutefois son intuition de base qui fait de l'expérience humaine (physique et/ou psychique) la source primordiale de l'expérience et de l'émotion esthétique. Ainsi, même au coeur de sa période la plus puriste, Fry réitère sa conviction de l'existence d'un lien fondamental entre l'art et la vie.

Ce rapport si important entre l'art et la vie, Fry le place cependant bien en dehors de l'utilitaire et de la vie pratique et active. Il fait intervenir ce qu'il décrit comme une double vie - la "vie imaginative"14 - qu'il pose dans un rapport d'écho (plus tard: reminiscences) avec la vie actuelle ${ }^{15}$. 
Il en parle ainsi en 1909 dans "An Essay in Aesthetics": man has the peculiar faculty of calling up again in his mind the echo of past experience [...], of going over it again, 'in imagination' as we say. He has, therefore, the possibility of a double life; one the actual life, the other the imaginative life. ${ }^{16}$

Pour Fry, cette double vie exige un certain détachement, mais s'alimente néanmoins à même les circonstances de la vie ordinaire et à même les sensations pour éveiller des feelings qui, dit-il, "semblent appartenir à certaines sources très profondes de notre nature" 17 . Essentiellement, Fry distingue la "vie imaginative" de "la vie actuelle" par le fait qu'elle soit exemptée des nécessités matérielles et des impératifs sociaux qui régissent la seconde. Cette "vie imaginative" serait "séparée de la vie actuelle par l'absence de réaction [responsive action]"18. L'observation ainsi libérée des contraintes de la vie pratique, pourrait alors s'ouvrir sur une vision plus complète, s'y attarder et découvrir "nombre de choses, très intéressantes bien que non-pertinentes, et qui, dans la vie réelle, n'atteindraient pas le niveau du conscient alors trop occupé à réagir à des problèmes immédiats" ${ }^{19}$. L'art serait, selon Fry, "une expression et un stimulus de cette vie imaginative" 2 .

L'expérience esthétique tient alors au fait que le sujet (producteur ou récepteur) veuille bien s'engager dans ce champ particulier et parallèle, et de là, à n'agir qu'à titre de spectateur détaché. L'art offre ainsi aux êtres humains la possibilité de s'observer eux-mêmes, d'observer leur environnement et de retrouver des émotions signifiantes, ordinairement bloquées par les nécessités d'ordre vital ou social. La transcendance divine et la métaphysique traditionnelle n'entrent pas en jeu dans cette expérience esthétique qui s'adresse - exclusivement et sans s'en excuser - aux phénomènes immanents à la nature et à l'expérience humaine $\mathrm{A}$ quelques variantes, hésitations ou suspensions près, cet axiome reste dominant et revient comme un leitmotiv à travers l'ensemble des textes de Fry qui, jusque dans ses Last Lectures, maintient que l'art est "une affaire de famille entre les êtres humains"21.

S'il fallait appuyer sur la persistance de Fry à associer le matériel et le spirituel, il suffirait de considérer les définitions du spirituel et de la vie spirituelle qu'il propose en 1933. Selon lui, "notre vie spirituelle" est constituée par:

those parts of the subconscious being which have filtered down through our conscious life and consist of the abiding residue of innumerable sensations, feelings, predilections, aspirations, desires, judgments, [...]. ${ }^{22}$

Puis il précise:
I use 'spiritual' throughout in a rather special, but I think necessary, sense. I mean by it all those human faculties and activities which are over and above our mere existence as living organisms. Used in this sense it avoids any prejudgment of what parcticular faculties come into play, whether intellectual, affectional or what not [...] But it is evident to anyone who reflects on human life that our biological needs are so insistent and overwhelming that no activity is likely altogether to escape their influence, and I think it is evident that works of art are no exception to this. ${ }^{23}$

Ces tressages du physique, du psychique et de l'intellectuel, qu'il rassemble sous la catégorie "du spirituel", réitèrent le lien déjà posé, en 1909, entre les sensations (physiques et physiologiques) et les émotions esthétiques ${ }^{24}$, et confirment chez Fry la persistance de cette tendance d'ordre phénoménologique.

Bell, toujours familier avec les positions de Fry ${ }^{25}$, agit en toute connaissance de cause lorsque, dans $\operatorname{Art}$ (1914), il se campe dans une position tout à l'opposé en déclarant:

To appreciate a work of art we need bring with us nothing from life, no knowledge of its ideas and affairs, no familiarity with its emotions. Art transports us from the world of man's activity to a world of aesthetic exaltation. For a moment we are shut off from human interests; our anticipations and memories are arrested; [...] he who contemplates a work of art inhabits a world with an intense and peculiar significance of its own; that significance is unrelated to the significance of life. In this world the emotions of life find no place. It is a world with emotions of its own. ${ }^{26}$

Il ne s'agit donc pas, pour Bell, de se détacher d'un ou de quelques aspects seulement de la vie. Bien au contraire, il parle expressément de s'en détourner tout à fait pour prendre contact avec un autre monde - étranger et indépendant - où il connaîtrait une "signifiance" sans aucun rapport (unrelated) avec la signifiance liée à la vie. Bien évidemment, l'attachement et l'intérêt que Fry manifeste à l'égard du phénoménal et de ses complexités, n'atteignent aucunement Bell qui, pour sa part, tend vers un ailleurs nouménal lui permettant d'oublier les contingences de l'espace et du temps, de même que les vicissitudes humaines qui l'agaçent $\tan \mathrm{t}^{27}$. Ses inquiétudes et ses attentes ressortent clairement lorsqu'il ajoute:

The ideas of men go buzz and die like gnats; men change their institutions and their customs as they change their coats; the intellectual triumphs of one age are the follies of another; only great art remains stable and unobscure because the feelings that it awakens are independent of time and place, because its kingdom is not of this world. ${ }^{28}$ 
Cette proclamation de l'existence d'un autre monde, auquel l'art appartiendrait exclusivement, relève d'une tendance qui sera confirmée dans plusieurs textes ultérieurs. Dans Since Cézanne, publié en 1922, Bell reprend la même conception de l'expérience esthétique. Il écrit: "ce que nous ressentons n'a rien à voir avec une humeur préalable; nous sommes transportés dans un monde blanchi de toute expérience passée, esthétique ou sentimentale" ${ }^{29}$. Il s'agit toujours d'un univers voué à "l'admiration impersonnelle et désintéressée" ${ }^{30}$. Puis, dans Enjoying Pictures, parus douze ans plus tard, il reprend la même hypothèse à savoir que:

\section{Both ['artist' and 'aesthete'] are able to rise on the wings of art to a world above the world of normal experience. [...] From this world of the spirit they look down on the world of our sorrow. ${ }^{31}$}

Ainsi que son corollaire — négatif-à l'effet que:

\begin{abstract}
It is not to be supposed that the aesthete or anyone else spends all his waking hours in that world. Even of art the wings tire. We all return to earth and waste a good part of our lives there. ${ }^{32}$
\end{abstract}

Dans cet esprit - à l'opposé de Fry, mais en accord avec le credo hégélien - c'est à la métaphysique, au religieux, à l'absolu, bref à ce qui transcende la nature et la condition humaines, que Bell relie l'autre monde dans lequel il se dit transporté par ses expériences esthétiques ${ }^{33}$.

L'enchevêtrement physique, physiologique, psychique, intellectuel et spirituel, si important pour Fry, n'évoque chez Bell qu'une promiscuité déplorable, voire répugnante. En tirant ainsi une frontière étanche entre le matériel et le spirituel, et en isolant de part et d'autre de cette frontière l'expérience esthétique de l'expérience humaine, Bell rejoint l'aile radicale de l'esthétique néo-platonicienne (et hégélienne) que nous évoquions plus tôt.

\section{CONDITIONS ET MOYENS D'ACCES A L'EXPÉRIENCE ESTHÉTIQUE}

La définition des conditions et des moyens d'accès à l'expérience esthétique suppose d'autres points de divergence entre Fry et Bell. De manière générale, Fry considère l'expérience esthétique comme accessible à tous les êtres humains (au moins en ce qui concerne la réception de l'oeuvre). Il insiste cependant sur la médiation obligatoire d'un processus formateur dans lequel le spectateur devra s'engager activement, et sans lequel l'aspect esthétique restera stérile. Les moyens que Fry désigne comme pouvant conduire à l'expérience esthétique - imagination (echoes, reminiscences) ou subconscient - se trouvent aussi sévère- ment circonscrits par cette contrainte éducative. Pour Bell, l'accès serait beaucoup plus simple quoique beaucoup plus restreint. En effet, il suffirait d'être doué d'une disposition innée ("sensibilité esthétique") pour parvenir, sans les contraintes et les tracasseries d'un apprentissage, à l'essentiel de l'expérience esthétique. Il s'agit donc, d'une part, de conditions et de moyens universels dont l'actualisation dépend de la volonté et de l'action du sujet, et d'autre part, de conditions et de moyens exceptionnels qui pour l'essentiel s'exercent en dehors de la volonté et de l'action du sujet.

Lorsque Fry parle de l'imagination et de son contenu d'echoes dans "An Essay in Aesthetics", paru en 1909, ou du subconscious et de son contenu de reminiscences, dans "ArtHistory as an Academic Study", publié en 1933, il envisage toujours des canaux qui s'écartent de la conscience actuelle et entraînent le sujet vers le non-conscient. Cela, combiné avec une utilisation fréquente du mot unconscious, laisserait entendre un intérêt pour l'inconscient freudien et pour des recherches et des interprétations d'ordre psychanalytique. Mais est-ce bien le cas? La réponse à cette question s'avère capitale puisqu'elle détermine les créneaux de sens imputables ou non à son expression significant and expressive form. Dans un premier temps, Fry pose l'imagination comme une faculté - donc, comme une entité agissante qu'il présente aussi comme spontanément constructive ${ }^{34}$. A ce dispositif structural, Fry ajoute un potentiel langagier en évoquant un "langage visuel de l'imagination" et un "langage de la forme et de la couleur" 35 - que Cézanne et les post-impressionnistes auraient en partie redécouvert. Conséquemment, Fry demande à l'oeuvre d'art de témoigner de l'actualisation par l'artiste de ce potentiel constructif et langagier (l'artiste doit réussir à construire, à articuler, à "dire quelque chose" 36 dans les termes de ce langage visuel), et, parallèlement, à ouvrir des brèches et d'inviter la participation active et personnelle de l'imagination du récepteur ${ }^{37}$. L'ensemble des textes de Fry insiste sur cette nécessité de "dire" et de "lire". Jusque-là, il n'y a pas d'antinomie entre l'imagination selon Fry et l'inconscient auquel Freud attribue aussi une logique et une dynamique langagières. Cependant, les mises en garde énoncées par Fry en rapport avec les dangers inhérents à l'accès à la vie imaginative, signalent une incompatibilité avec le projet psychanalytique qui apparaît bientôt comme incontournable. En effet, malgré sa présentation de l'imagination comme lieu de liberté (par rapport à la vie pratique), Fry ne tarde pas à circonscrire cette liberté, et ce sur plusieurs plans, sur le plan des comportements, sur le plan des thèmes et des moyens d'exploration. En ce qui concerne les comportements, Fry déplore le fait que la libération de toute nécessité de réaction appropriée, entraîne généralement une insouciance qui va 
jusqu'à l'impertinence (emotion of self-aggrandisement ${ }^{38}$ ). Il réprouve cette attitude "infantile" 39 et veut qu'elle soit corrigée de manière à ce que:

\section{[...] with the teaching of experience and the growth of char- acter the imaginative life comes to respond to other instincts and to satisfy other desires, until, indeed, it reflects the high- est aspirations and the deepest aversions of which human nature is capable. 40}

L'expression, issue du niveau infantile de l'imagination celle d'un moi (self) impertinent, péremptoire ou tout simplement centré sur lui-même (self-aggrandisement, selfassertive, self-conscious, self-important, self-satisfied, etc.), constitue donc un objet de censure plutôt qu'un objet d'étude. De manière générale dans les textes de Fry, les références à la petite enfance, qu'il s'agisse des "extravagant romances" de l'imagination infantile 41 ou des "meaningless mosaic of flat patches of colour" par lesquelles la vision impressioniste rejoint la naïveté du nouveau-né ${ }^{42}$, restent péjoratives et suggèrent un stade à dépasser plutôt qu'à revoir et à étudier. Cette répression du moi (self) infantile et, à l'opposé, l'approbation d'un moi (self) devenu humble, modeste et attentif à ce qui lui est extérieur, s'énonce de manière particulièrement appuyé dans Cézanne - A Study of His Development. Même en dehors du cas de Cézanne, cette prescription de la part de Fry s'affirme de manière soutenue et s'étend jusqu'au rejet de toute expression perçue comme provenant de visions intérieures (inner visions), et jusqu'à la valorisation exclusive d'une expression orientée et articulée en rétroaction entre le sujet et ce qui lui est extérieur - "la chose vue" (the thing seen). Tout un ensemble de thèmes introspectifs se trouvent ainsi exclus de l'expression esthétique jugée signifiante.

Une variante de ce dualisme se profile aussi sous l'affrontement perçu entre deux inclinations innées. La première - négative et introvertie - agirait à partir de particularités et d'ambitions personnelles, soutenues par des idées ou des modèles pré-existants suggérés par la culture, par exemple, le romantisme débridé de Cézanne et son attitude étrange à l'égard des femmes, qui le poussent vers des contortions et des involutions baroques ou des représentations d'un érotisme torturé et déconcertant ${ }^{43}$. La deuxième variante - cette fois positive - permet une capacité exceptionnelle de voir et d'exprimer un rapport singulier avec le monde extérieur ${ }^{44}$; elle représente la véritable nature-instinct de l'artiste ${ }^{45}$, par exemple, "l'instinct plastique inné [de Courber]", "la manière instinctive par laquelle [Duccio] donne de telles différences de poids et de densité à sa variété de couleurs locales" ou encore "la sensibilité chromatique de Cézanne, "Cézannés chromatic sensibility", etc. ${ }^{46}$. Chez le récepteur, cette ouver- ture sur l'extérieur se manifeste par une participation active visant à dépasser le stade du sentimentalisme ou de la première impression de "like or dislike" ${ }^{7}$. Cependant, vu que la première inclination s'exprime plus facilement, plus spontanément, et tend à dominer la seconde, il importe de trouver un renfort qui privilégie le développement et la prédominance de l'inclination positive. Cet appui consiste en l'apprentissage d'une méthode rigoureuse qui canalise l'expression et raffine la réception. Pensons, par exemple, dans le cas de l'artiste, à la méthode de Pissarro, d'abord suivie à la lettre par Cézanne, puis reconstruite pour servir la spécificité de sa propre vision ${ }^{48}$; et dans le cas du spectateur, à la méthode utilisée par Fry lui-même et transmise dans ses Slade Lectures $^{49}$. Les textes de Transformations, Cézanne - A Study of His Development ou Henri-Matisse, donnent plusieurs exemples de l'incompatibilité fondamentale que Fry voit entre ces deux inclinations et de son affirmation sur la nécessité de choisir entre l'une ou l'autre, plutôt que de reconnaître la co-existence des deux. La prescription de Fry s'énonce clairement lorsqu'il applaudit, chez Cézanne et Flaubert, la répression de la tendance romantique au profit de la tendance classique ${ }^{50}$.

Fry discrimine tout aussi radicalement entre les moyens d'exploration jugés adéquats et ceux qui doivent être évités. Dans "An Essay in Aesthetics", il fait valoir que:

In dreams and when under the influence of drugs the im-
aginative life passes out of our own control, and in such
cases its experiences may be highly undesirable, but when-
ever it remains under our own control it must always be
on the whole a desirable life. 51 Il poursuit en assignant à l'art un rôle d'agent régulateur: " $A r t$, then is, if I am right, the chief organ of the imaginative life; it is by art that it is stimulated and controlled within us" 52 .

Toutes ces restrictions et le désir de contrôle qui les traverse, montrent un écart considérable entre Fry et la psychanalyse, du moins telle qu'il la percevait à l'époque. Il s'ensuit que les mots et les notions de unconscious ou de subconscious, lorsqu'ils apparaissent de manière favorable dans les textes de Fry, ne font assurément pas référence à l'inconscient freudien ou à sa charge de désirs et de représentations primaires - infantiles - véhiculée principalement par les rêves. D'ailleurs, à l'encontre de Herbert Read et d'une théorie favorable à la méthode surréaliste en général et aux oeuvres de Dali en particulier, Fry déclare expressément son refus de reconnaître tout aspect raisonnable à l'inconscient profond, et repousse toute possibilité d'un contact fructueux entre le niveau de l'inconscient profond et celui du pré-conscient. Il marque ainsi la frontière de l'aventure qu'il propose en dehors du rationnel et du conscient: 
In fact, the worship of the lowest, most unreasonable levels of the unconscious life which underlies so many of the methods of modern art is absurd. It is only in those higher levels which come into close contact with our conscious intellectual and organized life that the fruitful synthesis both of science and art occur. ${ }^{53}$

Il continue en réitérant sa position fondamentale qui rejette l'introspection et l'irrationnel au profit d'une vision balisée et orientée vers le monde extérieur:

\section{[...] moreover the desire to tap the unconscious which in-} spires the efforts at intuitive unreason of these artists, actually defeats its own ends. It is only when we concentrate on some external problem that the higher levels of our unconscious cerebration offer us significant synthetical results. ${ }^{54}$

De cette manière, tant sous la rubrique de l'unconscious ou du subconscious que sous celle de l'imagination, Fry clôture très strictement l'ouverture sur le non-conscient. C'est sous cet éclairage qu'il convient donc de voir ses déclarations à l'effet que l'art témoigne des "sources très profondes de notre nature", des "aversions les plus profondes", des "réminiscences immensément généralisées, très profondes et très vagues", ou encore des "vérités les plus profondes" 55 , et de retenir que, pour Fry, les vérités profondes qui concernent l'art n'atteignent jamais les grandes profondeurs de l'inconscient freudien mais s'arrêtent plutôt au niveau que Freud définit comme celui du pré-conscient ${ }^{56}$. En 1933-4, dans "Art-History as an Academic Study", Fry se dit toujours dans l'attente de recherches en psychanalyse qui dépasseraient le stade du mapping out de l'inconscient et des "modèles émotifs inscrits durant les premières années de l'enfance" 57 . Ces recherches, ajoute-t-il, pourraient bien un jour s'avérer pertinentes quant à la nature de la création artistique, mais il s'en détourne, une fois de plus, en faveur d'une concentration sur le niveau "pré-conscient (subconscious) $)^{58}$.

Fry utilise aussi le mot et la notion d'unconscious d'une autre manière, qui concerne également notre propos. Il s'agit alors d'une référence à un mécanisme nerveux et/ou physiologique - donc indépendant de la volonté du sujet, et qui témoignerait de manière directe, soit de sa "condition nerveuse habituelle", soit de son "humeur" au moment où le geste est fait. C'est le domaine des rythmes vitaux, du handwriting - un domaine que Fry retient comme pertinent, important même, en ce qui concerne l'expression esthétique. Même sur ce plan où l'expression relève spécifiquement du sensible plutôt que de l'intelligible, Fry cherche encore une marque de contrôle - un "contrôle [du] nerveux" 59 . Mais puisqu'il doit reconnaître que ce niveau d'expression ne se manifeste qu'en dehors des cadres du conscient, il admet alors un certrain retrait de la conscience. Ce retrait ne sera toutefois que partiel, juste suffisant ${ }^{60}$, semble-t-il, pour laisser passer une dose convenable de vitalité et de sensibilitét ${ }^{\text {. }}$. C'est, en quelque sorte, comme si le conscient était invité à tourner le dos sans toutefois quitter la pièce.

Ainsi, chacune des voies ou des niveaux que Fry ouvre sur le non-conscient se trouve balisée et surveillée par le conscient. La liberté initialement associée à la vie imaginative, cède partout la préséance à la réalisation d'un double impératif: celui du pouvoir-voir, et celui du devoir-dire (en ce qui concerne l'artiste) ou devoir-lire (en ce qui concerne le spectateur). Bref, la vision ouverte, élargie sur un nouveau champ est aussitôt soumise à une entreprise d'articulation qui devra "dire" ${ }^{62}$, mais pas tout ou n'importe quoi et certainement pas n'importe comment.

Clive Bell, pour sa part, n'a pas à s'engager dans de telles précisions. En accord avec son premier axiome qui exclut toute liaison entre l'expérience esthétique et l'expérience humaine, il ne s'attarde guère sur ces dispositifs humains que sont l'imagination, le subconscient, les rythmes vitaux, etc. Il invoque plutôt une faculé surhumaine - un don, une "sensibilité particulière" - qui le rendrait apte à capter l'émotion esthétique directement et immédiatement. Cette condition - unique, simple et implacablement élitiste - Bell la pose et la répète depuis $A r t$ jusqu'à Enjoying Pictures. En voici quelques manifestations: "a great gift of aesthetic appreciation ... at least as rare in visual as in musical art". Ou bien: "Only those who have been born with a peculiar sensibility, and have known how to cherish it, enjoy art naturally, simply, and at first hand as most of us enjoy eating, drinking and kissing". "They [the "wings of art"] sprout on those only who have been born with a peculiar sensibility, without which neither general education nor intensive culture will make them grow an inch" 63. Il avance même des chiffres pour soutenir son idée: "Sur cent mille personnes, neuf cent quatre-vingt-dix-neuf mille neuf cent quatre-vingt-dixneuf naissent privées de sens esthétique", déclare-t-il dans "The Colonel's Theory"(1925)" 64 .

Contrairement aux moyens préconisés par Fry, des moyens axés sur le "dire" et sur un processus de rétroaction entre le sujet et l'objet, les moyens prônés par Bell ne sollicitent en rien les facultés analytiques ou dialectiques de l'esthète. Puisque Bell pose l'essentiel de son expérience esthétique dans le domaine de l'"ineffable", de l'indicible, un domaine où les capacités articulatoires du sujet se trouvent court-circuitées, c'est donc en dehors de tout effort ou volonté que l'esthète reçoit la quintessence de ce que l'art a à offrir. Déjà dans $A r t$, en prenant appui sur le Principia Ethica de G.E. Moore ${ }^{65}$, Bell affirmait que: "no quality can be defined" 66 , et, puisqu'il avait déjà présenté sa Significant Form comme une qualité ${ }^{67}$, le couple Significant 
Form-aesthetic emotion se trouvait, d'entrée de jeu, mis à l'abri de toute tentative d'analyse, de description ou de définition. C'est cette vision que nous retrouvons vingt ans plus tard dans Enjoying Pictures lorsque Bell décrit le moment crucial de son expérience esthétique:

\section{[...] [the aesthete] talks aloud, much as children prattle at play, not greatly caring whether they are heard or under- stood. At first maybe he talks a little wildly, so that what he says is not what he wants to say: the fact being that what he feels, and tries to express at first, at the first im- pact of the whole work, is nothing less than the aesthetic thrill - a feeling ineffable. ${ }^{68}$}

Cet état d'excitation ineffable constitue aussi le point culminant de l'expérience du critique-esthète telle que décrite dans Since Cézanne:

No one can state in words just what he feels about a work of art - especially about a work of visual art. He may exclaim [...] He may go on to seek some rough equivalent in words for his excited feelings. But whatever he may say will amount to little more than steam let off. He cannot describe his feelings; he can only make it clear that he has them. That is why analytical criticism of painting and music is always beside the mark [...] before a work of art the critic can do little more than jump for joy. ${ }^{69}$

Ainsi, le plaisir immédiat que Bell ressent dans l'inarticulé (déclaré inarticulable) contraste fortement avec celui que Fry éprouve en cherchant, par la médiation de facultés analytiques, à tirer l'inarticulé vers l'articulable. Les conditions et les moyens d'accès soutenus, de part et d'autre, reflètent fidèlement ce contraste. Pour ce qui est de l'offensive commune contre le rationnel, Bell, en invoquant l'Irrationnel mystique ou religieux, s'avance bien au-delà de Fry qui, pour sa part, refuse expressément de s'aventurer dans ce domaine: "On the edge of that gulf I stop", écrit-il, en 1920 , à propos des explications ouvrant sur le mysticisme ${ }^{70}$. La signifiance entrevue par Fry sera donc d'un type apte à transiter par l'intellect et relèvera d'un non-rationnel rationalisable, alors que celle proclamée par Bell restera à la fois plus libre et irréductiblement nébuleuse.

\section{MANIFESTATIONS DE L'EXPÉRIENCE ESTHÉTIQUE: ÉMOTION(S), "FEELINGS"}

Au sens strict ou dans une acceptation plus large, l'émotion relève de phénomènes affectifs et implique un ébranlement - plus ou moins brusque, violent ou intense - de "l'ensemble de notre état de conscience" "1 . Le rôle primordial accordé à l'émotion comme la manifestation d'une ex- périence esthétique accomplie, constitue chez Fry et chez Bell, une autre expression du désir d'une distanciation, plus ou moins importante, par rapport au rationnel et au conscient. Mais, d'entrée de jeu, les deux auteurs appliquent une censure qui, en accord avec l'esthétique traditionnelle, n'admet que les émotions positives; celles, qui appartiennent à la catégorie des "maladies de l'âme"72 (Kant) ou à celle du refoulement (Freud), se retrouvent effectivement refoulées. Fry le dit expressément, en 1912, lorsqu'il réfute les théories et les oeuvres futuristes en soutenant que l'esthétique ne peut dépendre que d'une émotion "de type positif, qui s'apparente davantage à l'amour qu'à la haine"73. Vingt et un ans plus tard, en 1933, il persiste en écartant les émotions, "très étranges et inquiétantes", dévoilées par la psychanalyse $^{74}$. Bell, pareillement, restreint son esthétique à une disposition émotionnelle positive. Mais, une fois de plus, au-delà de ce point d'entente, les divergences s'affirment nettement en ce qui concerne la nature, l'unicité et l'intensité de ces émotions positives mises en cause.

D'Art (1914) à Enjoying Pictures (1934), le profil de l'émotion esthétique évoquée par Bell ne change guère. Il s'agit toujours d'une émotion aussi ultime que la "réalité ultime" qui s'y manifeste ${ }^{75}$, une émotion de nature intemporelle et absolue ${ }^{76}$, une émotion détachée et isolée de l'humain. Bell en parle en ces termes: "Some profound and general emotion common, or at least possible, to all ages, and peculiar to none"; "[A] grand aesthetic thrill [which] is absolute; "[an emotion] different from and transcending the emotions of life, transporting men to ultra-human ecstasies"; "[A] remote aesthetic beatitude, in which, freed from humanity, we are upstayed by [...] pure visual form"; "Ultra-human emotions"77. De plus, conformément à la manière syncrétique dont elle surgit, cette émotion apparaît comme unique, globale et irréductible. Dans Art, Bell la présente comme: "une émotion singulière [...] une sorte particulière d'émotion provoquée par les oeuvres d'art visuel"78, mais, précise-t-il: "Je ne veux pas dire, bien sûr, que toutes les oeuvres provoquent la même émotion. Au contraire, chaque oeuvre produit une émotion différente. Mais toutes ces émotions sont, à l'évidence, de la même espèce"79. Plus tard, dans Enjoying Pictures entre autres, même lorsqu'il l'entoure de plaisirs accessoires happiness, variety of pleasures, subsidiary pleasures, etc. $\left.{ }^{80}\right)$, cette émotion particulière demeure toujours irréductible et inaccessible à l'analyse. Ce statut exceptionnel s'étend au canal sensoriel par lequel cette émotion transite. Ainsi, même si Bell remarque à plusieurs reprises que l'émotion esthétique doit passer par les yeux, il la dégage néanmoins de toute influence d'ordre sensoriel en disant:

All emotion, says the common man of science, must ultimately be traced to the senses. All moral, religious and aes- 
thetic emotions are derived from physical needs [...] 'Not so' reply saints, artists, Cambridge rationalists, and all the better sort; for they feel that their religious, aesthetic, or moral emotions are not conditioned, directly or indirectly, by physical needs, nor indeed, by anything in the physical universe. [...] There are things in life the worth of which cannot be related to the physical universe, - things of which the worth is not relative but absolute. ${ }^{81}$

Le credo proclamé dans Art concorde avec cette pratique, habituelle chez Bell, qui consiste à cloisonner de manière étanche des domaines contigus que Fry, pour sa part, pose plutôt en interaction. Cela permet à Bell de postuler le canal visuel comme un véhicule idéal, abstrait du contexte et des contingences concrètes qui le constituent par ailleurs. Bien que cette désincarnation du visuel puisse sembler farfelue lorsque présentée dans les termes romantico-mystiques utilisés par Bell, elle s'apparente tout de même aux positions que tiendront d'autres critiques modernistes, défenseurs de l'abstraction, notamment Clement Greenberg et Michael Fried. Ce postulat s'accorde également avec des idées beaucoup plus anciennes, comme celles de l'abbé Suger qui, au XIIe siècle, sanctifiait le couple vision - claritas et le distinguait des autres affaires d'ordre sensoriel.

Outre ces qualificatifs qui concernent principalement la nature exceptionnelle de l'émotion esthétique, Bell nous offre une abondance de déclarations quant à l'intensité et à la durée de cette émotion. Par exemple, la réitération d'expressions telles que: "passionate emotion", "aesthetic ecstasy", "aesthetic exaltation", "an emotion more profound and far more sublime", "thrilling raptures", "pure aesthetic rapture", "aesthetic beatitude", "most sublime [...] most wonderful emotions", "emotional beatitude, "intensely delightful", "grand aesthetic ecstasies", "supreme rapture", "extraordinarily intense and desirable emotions", "grand aesthetic thrill" >etc. ${ }^{82}$ ne laisse aucun doute sur l'intensité extrême de l'affect mis en cause par Bell. Aussi, il décrit cette émotion comme étant très brève, brusque, inattendue et enlevante. Ces quelques formulations sont typiques: "the contemplative moment is short", "momentary shock", "sparse and to be measured generally in minutes or even seconds", "a thrill [...] short and sharp", "a sudden vision", "a sudden and violent reaction to the essence", "[a] peculiarly violent shock striking on native sensibility", "we are taken unawares and bowled over", "lifted above the stream of life", "carries a person [...] out of life into ecstasy", "carried [..] off my feet", "carried to the high regions of art", "transported into a world washed clean of all past experience", "that supreme rapture in which we are carried", "transported in an instant", "rise on the wings of art to a world above", "the thrill which transported $m e^{\text {"83. }}$. Ce type d'émotion correspond à la fois au sens étymologique du mot et à la définition proposée par Ribot, qui la voit comme: "un choc brusque, souvent violent, intense, avec augmentation ou arrêt des mouvements" 84 . De plus, lorsque Bell parle d'une: "confiance émotionnelle, [de l'] assurance du bien absolu, qui fait de la vie un ensemble important et harmonieux" ${ }^{85}$, il rejoint aussi Lalande qui dit de l'émotion qu'elle: "réalise une unité dans la vie de l'esprit, tous les états de conscience actuels étant pénétrés par l'émotion dominante" 86 .

Fry aborde l'aspect émotionnel de l'expérience esthétique à partir d'un tout autre registre, un registre qui l'éloigne considérablement de l'émotion telle que définie plus haut. Le niveau et le moment extatiques ne sont guère dans ses cordes, pas plus d'ailleurs que les spéculations sur la nature "ultime" de l'émotion dite esthétique. En réponse aux affirmations trouvées dans $A r t$, Fry écrit:

Mr. Bell expresses rather a pious belief than a reasoned conviction that the aesthetic emotion is indeed an emotion about ultimate reality, that it has, therefore, a claim as absolute as the religious emotion has upon those who feel it [...].

[...] I feel that the ultimate nature of aesthetic experience still eludes Mr. Bell and all of us, as indeed it may well do for some centuries to come. ${ }^{87}$

S'appuyant sur ce principe, la théorie et la critique de Fry évitent les déclarations irraisonnées et proposent plutôt un processus de questionnement et d'observation empirique qui suit attentivement les pistes prometteuses, mais acceptent aussi de rebrousser chemin ou de suspendre toute conclusion dans les cas d'impasse ${ }^{88}$. Il s'ensuit que le discours de Fry demeure plus complexe et plus nuancé, particulièrement en ce qui concerne l'analyse de l'"émotion". Cette notion lui pose plusieurs problèmes mais il lui accorde néanmoins une place importante, depuis "An Essay in Aesthetics" jusqu'aux Last Lectures, sous les appellations variées de: aesthetic feeling, aesthetic emotion, plastic feeling, feeling about form, feeling for significant proportions, ou, moins spécifiquement, feeling ou emotion" ${ }^{89}$. Dans "An Essay in Aesthetics", Fry trace un enchaînement ${ }^{90}$ où le sentiment (feeling) esthétique apparît comme l'aboutissement d'un processus déclenché au niveau sensoriel, puis réactivé, parallèlement, par un rappel qui est aussi, en partie, d'ordre sensoriel. Là se fonde l'aspect concret que Fry continuera d'exiger de l'oeuvre et de l'expérience esthétique ${ }^{91}$. Concurremment, Fry souligne que les sensations, aussi nécessaires qu'elles soient, ne portent que l'amorce de l'expérience esthétique. Ultimement, celle-ci doit se développer et s'accomplir à des niveaux moins primaires et par le truchement déterminant d'une instance structurante: perception, imagination, intelligence-sensible ou l'équivalent. Cette intervention produit une émotion mêlée de cognition et de 
signifiance: l'"entendement émotionnel" (emotional understanding ${ }^{92}$ ). Aussi, en 1920 dans "Retrospect", reproche-t-il aux "décorateurs" de n'avoir pas su "distinguer entre des sensations agréables et la signifiance imaginative" "3. Cette "signifiance imaginative" n'intervient qu'à partir d'un plan syntaxique (celui des arrangements, des relations), où la participation des sensations s'estompe. Mais l'atténuation ne va pas jusqu'à l'anéantissement dans l'idéal comme chez Bell. Au contraire, Fry retient et intègre positivement les sensations dans l'avènement et le déroulement de l'expérience esthétique. Il les montre entre autres comme agissant continuellement - en tant que lien avec le concret - pour poser et retenir l'expérience dans une logique différente de la logique strictement intellectuelle. Par exemple, en parlant de la texture d'une peinture, il écrit: "[...] these perpetual slight variations of surface keep the mind and attention fixed in the world of sensation. We are as it were, forced to abandon our intellectual in favour of our sensual logic" ". Fry s'écarte donc des: "saints, artists, Cambridge rationalists, and all the better sort" invoqués par Bell'95 et tend plutôt vers l'"homme de science" (common man of science) en insistant sur un rapport d'association plutôt que de dissociation.

C'est dans cet esprit que Fry établit des liens entre certaines composantes plastiques de l'oeuvre et les sentiments qu'elles suscitent. Dans la ligne, par exemple, - la qualité nerveuse et le rythme de la ligne à main levée - il perçoit la présence, la sensibilité et le geste de l'artiste. Ce premier contact d'ordre sensoriel déclenche déjà un premier niveau d'émotion par empathie. L'empathie croît et se raffine par la reconnaissance d'une attention particulière portée à l'organisation de l'oeuvre, à la syntaxe donc: "la perception d'un ordre et d'une variété délibérés" 96 . Fry l'interprète comme une autre trace de la présence et de la parole de l'homme (l'artiste) qui, délibérément, a cherché à l'émouvoir. Cette reconnaissance constitue, selon lui, une partie essentielle de l'expérience esthétique ${ }^{97}$. L'importance de l'empathie comme facteur d'émoi esthétique ne fléchit pas chez Fry, et dans ces dernières conférences, comme "Art-History as an Academic Study" ou "Sensibility", il réaffirme la nécessité de reconnaître dans l'oeuvre la présence de l'autre - un autre compatible, c'est-à-dire "one with whom we can communicate" 98 . Fry, touché par la trace graphique ou texturale, exigera de plus une structure syntaxique efficace et variée (order and variety), apte à refléter les "conditions de notre existence [...] dans le temps et dans l'espace" ou les "traces résiduelles laissées sur l'esprit par les différentes émotions de la vie" 99 . Dans ces conférences, Fry insiste toujours sur cet entrelacement de l'ordre et de la variété, de l'intellectuel et du sensible, du conscient et du non-conscient, qui compose l'art capable de l'émouvoir:
[. . .] design, planning, co-ordination of the parts in the whole corresponds more or less with our desire to find order in things, our sense of immutable law and causation. Sensibility, on the other hand, corresponds to our desire for variety, multiplicity, chance, the unforeseeable. We may say that the conscious mind tends to a mechanistic view of things, a view amenable to mathematical statement; the unconscious brings the vital element which eludes mathematical statement. ${ }^{100}$

Aussi, ajoute-t-il: “C'est dans cette région [...] qui s'étend entre l'ordre rigide et le chaos, que la sensibilité de l'artiste fonctionne"101. C'est aussi dans cette zone frontière, par la satisfaction de ces deux désirs et la collaboration de différents modes de préhension, que le sentiment (feeling) ou l'émotion esthétique de Fry se développent. Il s'agit donc d'un sentiment composite et évolutif qui contraste fortement avec le caractère pur et intemporel que Bell attribue à son "émotion esthétique".

Mais que penser alors de la sympathie manifestée par Fry à l'égard de l'hypothèse si différente proposée par Bell? Sans aucun doute, en 1914, Fry se montre intéressé par l'assertion de Bell quant à l'existence d'une émotion particulière et indépendente des émotions de la vie, mais en même temps, il l'enjoint de compléter et d'étayer cette théoorie ${ }^{102}$. En 1920, Fry répète encore son intérêt pour une recherche visant à "isoler le sentiment purement esthétique, de l'ensemble des sentiments qui peuvent accompagner ou qui accompagnent généralement le sentiment esthétique que nous éprouvons devant une oeuvre d'art"103. L'appel à plus de précision se trouve donc relancé, vers Bell d'une part, puisque ce dernier s'est déjà engagé dans cette voie, mais aussi vers les chercheurs en psychologie expérimentale ${ }^{104}$. Quant à sa propre recherche, Fry choisit plutôt de la concentrer sur ce qu'il nomme "les éléments esthétiques" et sur l'analyse détaillée des oeuvres ${ }^{105}$. Ses textes ultérieurs (du moins jusqu'à ceux de Last Lectures) témoignent d'une relative mise en suspens de la recherche portant spécifiquement sur l'émotion, au profit d'une concentration sur les éléments formels et plastiques des oeuvres.

A propos de la sympathie que Fry manifeste à l'égard de l'hypothèse de Bell, il importe aussi de remarquer les réticences durables qui la mitigent. Fry par exemple, questionne la valeur de cette émotion esthétique, si intangible et peu accessible à l'ensemble de l'humanité, et doute de la possibilité de l'actualiser ${ }^{106}$. Aussi, l'utilisation de la troisième personne - inhabituelle chez Fry - pour désigner les tenants de l'émotion purement esthétique (“...those who experience it feel it to have [...] infinite importance in their lives" ${ }^{107}$ ), signale une distanciation et donne à entendre que 
Fry reste étranger à ce genre d'émotion. Dans "Some Questions in Esthetics" (1926), il met un terme au scepticisme poli qu'il maintenait depuis " $A$ New Theory of Art" (1914) et déclare: "qu'il n'est pas nécessaire, en ce qui concerne notre propos, de créer l'hypothèse d'une falculté spécifique ou mystérieuse"108. Fry rejette alors expressément l'aura de mystère cultivée par Bell et ajoute, qu'en ce qui le concerne, il s'agit plutôt d'une "orientation spéciale de la conscience" et non d'une émotion ou d'une sorte d'émotion particulière. La distinction est importante puisqu'à partir d'une orientation spéciale de la conscience ("a special disposition of the mind, a special orientation of the consciousness" 109 ), tout comme à partir du champ de la vie imaginative ${ }^{110}$, nous pouvons considérer un nombre infini de choses et d'expériences de qualités et d'intensités variables, et en retirer une aussi grande variété d'émotions. A l'opposé, l'"émotion d'un genre particulier" (a particular kind of emotion) postulée par Bell, relève d'un processus d'uniformisation et de réduction des variables. De manière générale, Fry parle d'émotions au pluriel, - alors que Bell utilise le singulier. Certes, il y a quelques exceptions, surtout durant la période entre 1914 et le début des années '20 alors que Fry s'intéresse de plus près à l'hypothèse de Bell, mais même là, le plus grand nombre d'utilisations de la notion d'"émotion esthétique" renvoie à la théorie de Bell et ce n'est que rarement que Fry reprend cette notion pour son propre compte.

Somme toute, l'effet généralement évoqué par Fry au sujet de l'expérience esthétique s'accorde mal avec la définition stricte de l'émotion vue plus antérieurement. Par rapport à Bell, pour lequel la définition de l'"émotion choc" s'avère toujours pertinente, la tendance de Fry requiert que nous parlions plutôt d'"émotions-sentiments" (selon la distinction proposée par P. Malapert ${ }^{111}$ ). Mais plus pertinemment encore, nous voyons dans la tendance de Fry un exemple de ce que Jean Piaget pose comme l'indissociabilité des fonctions affectives et des fonctions cognitives dans la conduite concrète des individus ${ }^{112}$, et suggérons que, bien plus que la nature et l'importance relative de l'une ou l'autre de ces fonctions, c'est leur interaction, leur re-présentation et leur ré-activation dans et par l'oeuvre qui intéressent et émeuvent Fry. Par contre, pour revenir à Bell, et toujours selon les théories de Piaget, ce serait un "préjugé romantique", qui se vérifie d'ailleurs dans plusieurs autres aspects des textes de Bell, qui le porterait à croire en des "données immédiates, des sentiments innés et élaborés", alors que l'effet "sublime" qu'il décrit et nomme "émotion esthétique" relève plutôt d'un processus de construction ${ }^{113}$.

\section{FINALITÉS ATTRIBUÉES A L'EXPÉRIENCE ESTHÉTIQUE}

Les finalités invoquées par Bell et par Fry engagent leurs lecteurs dans des directions tout aussi différentes. Alors que Fry tente de mettre à jour un processus communicationnel encore mal connu, et qu'il entrevoit un outil épistémologique apte à élargir la connaissance de l'être humain, Bell cherche un "refuge" qui permet de couper toute communication inter ou intra-subjective.

Bell ne cherche aucunement à masquer les objectifs de fuite et d'isolement liés à son modèle d'expérience esthétique. Bien au contraire, de manière récurrente et à plusieurs années d'intervalle, il valorise l'art en tant qu'échappatoire - refuge idéal - procurant un abri contre la médiocrité et l'instabilité humaines. Cette position devient éminemment claire dans Art (1914), à travers des énoncés tels que: "Art et Religion sont [...] deux voies par lesquelles les hommes échappent au circonstantiel, vers l'extase", ou, "Ce n'est pas en tant que sanctuaires protégeant de la vie - sanctuaires dédiés au culte de l'émotion esthétique - [...] que ces musées et galeries deviennent nocives" 114 ; ou encore: "Parce que les émotions esthériques sont à l'extérieur et au-dessus de la vie, il est possible d'y trouver refuge contre la vie"115. Ce leitmotiv revient en force dans Enjoying Pictures (1934), porté par les mêmes mots et par une rhétorique tout aussi emphatique: "Ceux qui prennent l'art au sérieux sont ceux qui y trouvent une évasion de la vie"116, écrit-il encore.

L'esthétique se trouve donc isolée de l'humain. Mais qu'en est-il de l'artiste, du producteur humain? Bell l'exclut également en précisant que:

In pure aesthetics we have only to consider < <>our emotion] and its object: for the purposes of aesthetics we have no right, neither is there any necessity, to pry behind the object into the state of mind of him who made it. ${ }^{117}$

Le caractère exclusif de cette relation entre l'esthète et "son objet" ne s'atténue pas. Au contraire, vingt ans plus tard, Bell le reprend par le biais d'une analogie avec l'amour. Il prêche alors la supériorité de l'extase esthétique sur l'extase amoureuse:

Love, the most intense of all human experiences [...] is unluckily also the most unstable. It has the drawback of depending on somebody else [...] Certainty and permanence are the things we most desire in love, and the only certain thing about it is that it won't endure. Now the ecstasies of art have this immense advantage, they depend only on one human sensibility and an almost inexhaustible supply of inanimate objects. 118 
Son désir d'exclure l'autre s'étend donc jusqu'aux objets [oeuvres d'art], qui doivent être perçus comme "inanimés". Enfin seul, l'esthète selon Bell aspire à la stabilité, à la certitude et à la permanence.

Après avoir ainsi décrété l'exclusion des rapports sociaux, puis celle des relations inter-personnelles, l'expérience esthétique de Bell pourrait-elle porter sur des questions intra-subjectives? Peut-il, par exemple, s'agir d'une démarche d'éclaircissement des rapports entre les différentes instances de l'appareil psychique de l'esthète (les rapports touchant le physique étant exclus d'emblée comme nous l'avons vu plus tôt)? L'esthète cherche-t-il à mieux se connaître? De nombreuses déclarations de Bell disent tout le contraire. En effet, à propos du moment culminant de l'expérience esthétique - l'"extase esthétique" ou le "grand frisson esthétique" - Bell célèbre plutôt un effet de dissolution du moi (self). Dans Art, il exalte: "ces instants où je me perds dans cet état infiniment sublime où la forme visuelle pure me transporte"119, et conclut : "Celui qui s'est déjà perdu dans un "O Altitudo", [...] Celui qui peut se retirer du monde jusqu'à l'extase saura quoi penser du circonstantiel"120. Dans Since Cézanne (1922), Bell glorifie: "ce ravissement suprême par lequel nous sommes transportés vers un monde d'admiration impersonnelle et désintéressée" "21. Il renchérit dans Enjoying Pictures (1934) en affirmant: "Ceux qui prennent l'art au sérieux [...] sont transportés hors d'eux-mêmes, hors de ce monde, dans le monde de l'esprit" ${ }^{122}$. La notion de connaissance de soi s'avère donc tout à fait incongrue en rapport avec une expérience qui produit un tel anéantissement du moi, ou un tel emportement hors de la conscience du moi. Ainsi, l'exclusion déclarée de la composante humaine peur s'étendre jusqu'à l'exclusion de la propre personne de l'esthète (impersonal, etc.).

L'amoindrissement de la composante humaine verse dans le pathos lorsque Bell s'attendrit en ces termes sur le saint ou sur l'artiste qui préfere les sévices, voire la mort, à tout compromis affectant son entreprise spirituelle, ou esthétique, ce qui revient au même:

I call him a religious man who, feeling with conviction that some things are good in themselves, and that physical existence is not amongst them, pursues, at the expense of physical existence, that which appears to him good. All those who hold with uncompromising sincerity that spiritual is more important than material life, are, in my sense, religious. For instance, in Paris I have seen young painters, penniless, half-fed, unwarmed, ill-clothed, their women and children in no better case, working all day in feverish ecstasy at unsaleable pictures, and quite possibly they would have killed or wounded anyone who suggested a compromise with the market. ${ }^{123}$
Toutes ces déclarations, en harmonie avec l'ensemble du discours de Bell, concordent remarquablement bien avec une vision schopenhauerienne qui pose l'art comme moyen de "libération du vouloir-vivre" 124 . Si bien que les quelques phrases suivantes, empruntées à Schopenhauer, s'harmonisent tout à fait à l'ensemble de l'esthétique prônée par Bell:

[...] du moment [dit Schopenhauer] qu'on se perd dans cet objet, [...] du moment qu'on oublie son individu, sa volonté et qu'on ne subsiste que comme sujet pur, comme clair miroir de l'objet [...] celui qui est ravi dans cette contemplation n'est plus un individu (car l'individu s'est anéanti dans cette contemplation même), c'est le sujet connaissant pur, affranchi de la volonté, de la douleur du temps. ${ }^{125}$

Mais, ajoute-t-il, alors que:

[...] "l'essence" de la vie, la volonté, l'existence elle-même est une douleur constante, tantôt lamentable et tantôt terrible [...] cette connaissance pure [la représentation pure dans les oeuvres d'arr] [...] ne l'affranchit pas définitivement de la vie, elle ne l'en délivre que pour quelques instants bien courts: ce n'est pas encore la voie qui mêne hors de la vie. ${ }^{126}$

Pour Schopenhauer, cette "voie qui mène hors de la vie" c'est la sainteté - l'état atteint par ceux qui: "n'attendent plus qu'une chose, c'est de voir la dernière trace de cette volonté s'anéantir avec le corps même qu'elle anime"127. Sur ce point encore, par les nombreux rapprochements qu'il fait entre les artistes et les saints, Bell contribue à engager l'art sur cette voie ultime indiquée par Schopenhauer.

Moins édifiante certes, mais tout aussi pertinente, la lecture psychanalytique-freudienne offre une autre interprétation des finalités déclarées par Bell. Freud, après avoir reconnu l'"état de détresse originel de l'être humain" et l'angoisse suscitée par les relations inter-humaines, postule une "expérience de satisfaction" par laquelle cette détresse se trouverait suspendue ${ }^{128}$. Cette expérience de satisfaction, liée au "principe de plaisir" et agissant selon un "processus primaire"129, ressemble fort à l'expérience esthétique de Bell. D'emblée, la détresse de Bell ne fait aucun doute. Pour se mettre à l'abri et hors de portée de la condition humaine, il cherche, indifféremment, un refuge, une évasion ou un sanctuaire; mais il veut aussi se prémunir contre les aléas des relations inter-humaines (même les meilleures dont il ne retient que les aspects négatifs: instabilité, incertitude, fugacité. Son expérience esthétique correspond alors à une "expérience de satisfaction" dans la mesure où elle suspend les causes de tension, en permettant un retrait (le sujet se trouve transporté dans un monde exempt de tension) et en promettant un es- 
pace et un temps de complète isolation du sujet avec "son objet" (le sujet jouit alors d'un accès exclusif et privilégié. Aussi, l'"objet", l'art en général, est présenté comme une entité bienveillante et puissante qui garantit la satisfaction totale, l'extase. Cette entité est stable, intarrissable ("inexhaustible supply of (...] objects") et vouée à une relation entièrement contrôlée par le sujet récepteur ("only our emotion and its object, depend only on one human sensibility"). Cette expérience donc, axée bien davantage sur le principe de plaisir que sur le principe de réalité, s'ancre dans un "processus primaire" qui - en dehors de tout effort d'adaptation de la part du sujet - promet une entière liberté, en même temps qu'une satisfaction instantanée et totale: la "béatitude" esthétique et émotionnelle. Cette interprétation explique pourquoi le moi (self) - en tant qu'instance inhibitrice du processus primaire ${ }^{130}$ - se trouve si nettement tenu à l'écart de la partie cruciale de l'expérience esthétique de Bell.

Au-delà de l'expérience esthétique, au-delà de la suspension du vouloir-vivre, ou "au-delà du principe de plaisir", Bell (dans la Réalité Ultime), Schopenhauer (dans la connaissance pure) et Freud (dans le Nirvana) se croisent dans la reconnaissance d'un désir - d'accès ou de retour - à un "océan de quiétude" 131 , posé en dehors de toute volonté et de toute individualité. Sur ce terrain, ils ne risquent pas de rencontrer Fry.

Ce dernier en effet, par sa persistance à soumettre et à évaluer l'art en fonction de ses qualités communicationnelles et cognitives, maintient son expérience esthétique à des niveaux, ou à des stades, bien différents de ceux cultivés par Bell. Déjà, en 1909, An Essay in Aesthetics expose plusieurs positions irritantes pour Bell. D'abord, Fry y déclare son admiration pour What is Art? (1898) de Tolstoï ${ }^{132}$ dont, malgré de nombreux désaccords, il retient néanmoins une idée - capitale pour lui - celle de: "l'art comme moyen de communiquer les émotions"133. Dans "Retrospect"(1920), Fry rappelle avec emphase l'importance de cette idée ainsi que tout le crédit dû à Tolstoï sur ce point particulier:

In my youth all speculations on aesthetic had revolved with wearisome persistence around the question of the nature of beauty [...] It was Tolstoy's genius that delivered us from this impasse, and I think that one may date from the appearance of What is Art? the beginning of fruitful speculation in aesthetic. [...] Tolstoy saw that the essence of art was that it was a means of communication between human beings. He conceived it to be par excellence the language of emotion. ${ }^{134}$

Dans "Art-History as an Academic Study"(1933), Fry réaffirme l'importance de l'art comme moyen de communication et il écrit l'Art. "is, I believe, the only means by which human beings can communicate to each other the quality and quiddity of their experiences [...] the work of art acts as a transmitting medium between the artist's subconscious nature and our own ...] the work of art [is] the central term - the liaison in a transaction which takes place between the artist and the spectator. 135

En plus d'insister sur la fonction communicationnelle, Fry précise le caractère cognitif et la portée à attribuer à cette communication. Il s'agit d'un approfondissement de la connaissance de soi - d'une partie du moi (self) jusque là latente et méconnue, et qui se verrait révélée par l'entremise de l'autre, un autre pourtant semblable: l'artiste. En 1909, Fry le l'écrit en ces termes: "We feel that [the artist] has expressed something which was latent in us all the time, but which we never realised, that he has revealed us to ourselves in revealing himself ${ }^{136}$. La communication concerne donc le domaine de l'inter-personnel, et elle se présente comme spéculaire. Ces deux caractéristiques subsistent dans "ArtHistory as an Academic Study", en des termes un peu plus précis. Fry désigne alors le niveau "subconscient" (de l'artiste et du spectateur) comme porteur de ces connaissances latentes qu'il s'agit de découvrir. Par contre, il reconnaît que les correspondances entre le subconscient de l'un et de l'autre n'apparaissent, ni dans tous les cas, ni facilement. Il pose tout de même ce que serait la communication idéale: "[il s'agirait d'un cas où l'artiste, ayant complètement incarné son exérience dans une oeuvre d'art, rejoindrait un spectateur apte à saisir parfaitement cette expérience"137. Il ajoute cependant:
[...] the message of a work of art is generally immensely complex, summarizing as I believe a whole mass of experi- ences hidden in the artist's subconsciousness. And this com- plexity renders it probable that each receiver only picks up a part of the total message. Therein lies the difficulty, and, considering the immense variety of receiving instruments that men possess, it is not surprising that they do not al- ways agree about the messages received. ${ }^{3} 8$

Il réduit alors le cadre spéculaire pragmatique aux cas où les principales composantes sont déjà compatibles et introduit cette condition déterminante: "s'il s'agit d'un artiste avec lequel nous pouvons communiquer"139. En dépit de ces aléas, Fry persiste à croire que l'art demeure: "le seul moyen par lequel les êtres humains peuvent se communiquer l'un à l'autre la qualité et la quiddité de leurs expériences" ${ }^{\prime 40}$. Il considère donc que l'art est porteur d'un potentiel cognitif relatif à la qualité et à l'essence de l'expérience humaine. Il soutient également que ce potentiel peut s'actualiser et faire l'objet de communications efficaces en- 
tre les individus. Ces communications favorisent alors un approfondissement de la connaissance de "soi" par chaque individu se trouvant ainsi mis en rapport - un rapport positif - à la fois avec l'"autre" et avec lui-même.

Dans la grille d'analyse freudienne, Fry, de toute évidence, s'inscrit à des stades bien différents. D'abord, au lieu de la détresse, c'est de l'intérêt et de la sympathie qu'il exprime vis-à-vis des êtres humains et des conditions dans lesquelles ils évoluent. Il favorise par conséquent un processus et une expérience de "liaison plutôt que d'isoement, et l'art lui apparaît comme ce "medium", ce facteur de rapprochement. Aussi, conformément aux caractéristiques que Freud assigne au "principe de réalité" et au "processus secondaire", l'expérience de Fry demeure liée à des efforts d'adaptation du sujet (intention, volonté, travail soutenu, etc.) ${ }^{141}$. De cette manière, la "voie la plus courte" et la plus immédiate privilégiée par Bell se trouve remplacée par une voie beaucoup plus longue. Le plaisir, moins intense mais aussi moins bref, s'échelonne progressivement du syncrétique à l'analytique $^{142}$ pour rejoindre néanmoins une source tout aussi intarissable ${ }^{143}$. L'objectif de renforcement et d'approfondissement du moi (self), soutient précisément le "moi-réalité" chargé de régir le processus secondaire et l'adaptation du sujet à la réalité extérieure ${ }^{144}$. Comme nous l'avons vu plus tôt, l'insistance de Fry à valoriser le regard orienté vers l'extérieur et à réprimer le moi $(s e l f)$ immature et centré sur lui-même confirme cet engagement dans un principe de réalité. Selon l'interprétation freudienne, cet engagement témoigne, positivement, de l'accès au stade de maturité de l'appareil psychique humain.

Selon la vision de Schopenhauer par contre, une telle utilisation de l'expérience esthétique rabaisserait Fry au stade du "vouloir vivre", le soumettant aux impératifs de la "Volonté>" et le maintenant à un échelon intermédiaire entre: "les degrés inférieurs [...] là où la volonté agit encore inconsciemment" et "les phénomènes multiples appropriés à la connaissance du sujet en tant qu'individu"145. En s'ancrant à ce niveau, Fry se priverait, volontairement, de la "connaissance spéciale" réservée à l'art, une connaissance qui "exige un oubli complet de la personnalité et de ses relations"146, et qui s'applique:

[...] à ce qui dans le monde subsiste en dehors et indépendamment de toute relation, à ce qui fait à proprement parler l'essence du monde et le substratum véritable des phénomènes, à ce qui est affranchi de tout changement et par suite connu avec une égale vérité pour tous les temps, en un mot aux Idées. ${ }^{147}$

En fait, lorsque Fry traite d'un même concept que Schopenhauer, comme par exemple la notion de substratum évo- quée dans "Pure and Impure Art" ${ }^{148}$, il lui donne une coloration ou lui adjoint une alternative d'ordre phénoménal (incluant le sensoriel) qui la détourne nettement d'une fixation sur l'éternel et l'universel. Bref, en termes schopenhauriens, Fry n'aurait pas bien saisi que: "le phénomène de la Volonté ["le vouloir-vivre"], l'univers, n'est essentiellement que douleur irrémédiable et misère infinie" 149.

Les divergences que nous venons de voir confirment amplement la déclaration initiale où Bell faisait état de ses désaccords profonds avec Fry. Mais surtout, ces différences établissent des paramètres de lecture et déterminent la portée et le sens attribuables aux notions de signifiance, de forme ou d'émoi, selon qu'ils apparaissent dans le cadre de l'esthétique de l'un ou de l'autre.

En ce qui a trait au développement de la théorie esthétique formaliste, Fry et Bell ouvrent donc deux catégories incompatibles. Bell soutient une esthétique platonisante qui s'allie à un credo métaphysique, immuable et intemporel, posé en dehors de toute relation avec l'expérience humaine concrète. Cette esthétique, qui postule une expérience purement subjective mais désincarnée, se déploie autour d'une "Forme" non-définie mais dite signifiante et dont la signifiance reste enfermée dans l'intime, bien à l'abri de toute nécessité d'éclaircissement ou de partage social. A la tradition néo-platonicienne et au palmarès des Idées Pures, Bell ajoute ainsi l'"Idée", l'appellation de "Significant Form". Cette appellation s'accorde bien avec certaines problématiques de l'esthétique moderne mais, en l'abandonnant à ce stade, Bell ne contribue guère à une recherche spécifique sur cette question. Tout cela fait de l'esthétique de Bell un lieu que nous dirons Réel ou fictif, un recours qui nous apparaîtra essentiel ou facile, selon nos options idéologiques. Fry choisit une démarche nettement plus pragmatique et spécifique qui, selon des méthodes d'ordre scientifique, propose d'engager une interaction entre l'esthétique et les autres sciences humaines, la psychologie, la psychanalyse,peut-être, l'anthropologie, etc., afin de préciser la signifiance portée par la forme plastique de l'oeuvre d'art. En dépit de l'immense complexité qu'il lui faut envisager, Fry croit valable d'étudier la significant and expressive form en relation avec le contexte humain dans lequel elle s'inscrit et de soumettre l'expérience esthétique - émotive, individuelle - à un processus d'examen, d'articulation et de partage social. Le formalisme de Fry s'apparente à la tendance actuelle préconisée par Louis Marin lorsque ce dernier parle d'un désir de "connaissance à visée scientifique de l'oeuvre d'art" ${ }^{150}$, qui prendrait soin de ne pas négliger la particularité du propos artistique. Aussi, lorsque Marin parle d'une "pratique du sens [...] indissolublement cognitive [sémiotique et sémantique], esthétique [sensible ou corporelle] et 
pathétique [émotionelle, affective, passionnelle]"151, nous retrouvons l'affirmation d'un entrelacement intime de l'intelligible (significant), du sensible (sensations) et de l'affectif (feelings, emotions), entrevu et esquissé par Fry. Ainsi, en dépit de certaines censures (émotions négatives, moi nonmature, inconscient profond) incompatibles avec une esthétique ou une sémiotique contemporaine, le formalisme de Fry offre néanmoins des ouvertures considérables qui le détachent de l'idéalisme éthéré de l'esthétique traditionnelle. De plus, en soumettant la signifiance à un agent extérieur au système formel (le " $x$ ", déterminant, qu'il ajoute à la Significant Form de Bell), Fry ouvre son formalisme sur une fonction sémiotique et le distingue d'un formalisme pur et dur qui se concentrerait exclusivement sur les spécificités internes du système formel.

* Je remercie madame Fernande Saint-Martin pour son intérêt à l'égard de mon projet de recherche et pour son appui soutenu. Je suis aussi redevable à Adele M. Ernstrom de m'avoir encouragé à publier ce texte et de m'avoir indiqué quelques sources nouvelles. Une partie importante de cette recherche a été rendue possible grâce à une aide financière du Fonds F.C.A.R.

1 L'expression est utilisée la première fois par Roger Fry dans son article "Post-Impressionism," publié dans la Fortnightly Review en mai 1911 et reproduit dans Post-Impressionists in England par J.B. Bullen, éditeur (New York, 1988), 179.

2 Les termes sont d'abord employés par Clive Bell dans "The English Group", Catalogue of the Second Post-Impressionist Exhibition en 1912, et repris dans Art en 1914.

3 Par exemple, J. B. Bullen dans l'introduction de son anthologie de textes, Post-Impressionists in England (New York, 1988), écrit que "though Bell's debt to Fry is enormous, their combined work in this field [formalist language] is a triumph of English empiricism" (p. 3) et Roberto Salvini dans l'introduction de Pure visibilité et formalisme dans la critique d'art au début du XXe siècle (Paris, 1988) note à propos de la théorie de l'art comme forme signifiante de Clive Bell: "la remarquable action qu'elle exerça sur un cercle de critiques d'art à l'esprit très brillant et dont se détache la figure très sensible de Roger Fry.

Le raisonnement de Clive Bell se développe selon cette méthode empirique et inductive qui est caractéristique, depuis $\mathrm{Ba}$ con, de la pensée anglaise" (p. 39). Le lien avec l'empirisme anglais, même s'il convient parfaitement à l'ensemble de l'oeuvre de Fry, ne peut s'appliquer qu'à un fragment notoire, mais non représentatif de la pensée globale de Bell - en l'occurrence les quelques phrases de "l'hypothèse esthétique" (Art, 7-11) où Bell annonce une éventuelle recherche empirique qu'il abandonne aussitôt au profit d'assertions quant au caractère essentiellement métaphysique de l'art et de l'émotion esthétique -. George T. Dickie souligne très clairement l'ambivalence de Bell concernant la nature empirique ou non (métaphysique), de sa "Significant Form", dans son article, "Clive Bell and the Method of Principia
Ethica," The British Journal of Aesthetics, 5, 2 (avril 1965), 139143. Par ailleurs, Simon Whatney assimile la pensée de Fry à celle de Bell et les accuse conjointement d'absolutisme (p.72), d'innéisme (pp. 70, 75 et 76), de solipsisme (p. 72), de mépris pour "le peuple" (p. 74), etc. Voir "The Connoisseur as Gourmet: The Aesthetics of Roger Fry and Clive Bell" in Formations of Pleasure (Londres, 1983). Ces concepts, bien qu'ils correspondent à l'attitude générale de Bell, n'ont rien de typique avec la pensée de Fry. Dans un échange entre Frances Spalding et Simon Whatley, celle-ci a beau insister sur la distinction à faire entre les idées de Fry et celles de Bell, (voir "Roger Fry and his critics in a post-modernist age," The Burlington Magazine, 128, 1000 (juillet 1986), 489-92), Whatney maintient Fry dans un rôle de puriste qui ne convient qu'à un moment de son cheminement intellectuel, (voir "Roger Fry and his friends in a postmodernist age: a reply to Dr Frances Spalding," The Burlington Magazine, 129 (avril 1987), 250), et là encore, toujours de manière mitigée. comme Spalding le souligne dans sa réponse à Whatney ("Simon Watney and his friends in a post-modernist age: a reply," The Burlington Magazine, 129 (avril 1987), 251). Selon le point de vue d'une histoire sociale de l'art, Victor Burgin rassemble Fry, Bell et Greenberg dans une démarche idéaliste allant de Platon à Greenberg. (Voir The End of Art Theory Criticism and Postmodernity (Atlantic Highlands, NJ, 1990), 11, 31, 67 et 147). C'est sans doute la distance idéologique qui empêche Burgin de voir que Fry n'a rien d'un platonicien et l'empêche de s'intéresser plus spécifiquement au fait que Fry s'oppose expressément à Bell en soutenant le caractère représentationnel de l'art (Voir "A New Theory of Art," Nation (7 mars 1914)], repris in Post-Impressionists (Bullen), 489-90) Par ailleurs, l'insistance de Fry sur la fonction communicationnelle de l'art l'absout de toute visée purement moderniste. Durant les années 1960, d'autres historiens ont aussi vu des alliances et des ressemblances là où nous voyons plutôt des divergences fondamentales. Nous pensons surtout à Eva Schaper qui parle d'une "Bell-Fry theory" pouvant se résumer par la formule "art is significant form" et se classer sous la rubrique de "radical formalism" (voir "Significant Form", The British Journal of Aesthetics, 1, 2 (mars 1961), 33-43); à Solomon Fishman qui considère l'esthétique de Fry comme étant très proche de celle de Bell (The Interpretation of Art, California, 1963); et enfin, Herbert Read qui relie Fry à Bell avant de les subsumer, indistinctement, sous une théorie déjà largement articulée par Vernon Lee, voir "Clive Bell" in The British Journal of Aesthetics, 5 , 2 (avril 1965), 107-110. Notre article propose un point de vue différent qui, sous certains aspects, rejoint ceux de David G. Taylor, voir son article,"The Aesthetic Theories of Roger Fry Reconsidered," The Journal of Aesthetics and Art Criticism, 36 (automne 1977), 63-72) et de Frances Spalding, voir "Roger Fry and his critics in a post-modernist age," The Burlington Magazine, 128, 1,000 (juillet 1986), 489-92.

4 De Roger Fry, les articles: "Giotto" (1900), 92-123; "An Essay in Aesthetics" (1909), 12-27; "The Art of the Bushmen" (1910), 609; "The Munich Exhibition of Mohammedan Art" (1910), 81- 
91; "The French Post-Impressionists" (1912), 166-70; "Paul Cézanne"(1917), 179-185; "The Artist's Vision" (1919), 33-8; "Negro Sculpture" (1920), 70-3; "El Greco" (1920), 143-8; "Retrospect"(1920), 199-212; repris dans Vision \& Design [1920] (Toronto, 1981) et les textes "Some Questions in Esthetics", 1-57; "Vincent Van Gogh", 235-248; "Seurat", 249-260; "On Some Modern Drawings", 261-281; "Plastic Colour", 282-297; "Appendix", 298-9, publiés dans Transformations [1926] (New York, 1956). En outre, Cézanne - A Study of His Development [1927] (Chicago, 1989). Henri-Matisse (Londres, 1930). Les conférences suivantes: "Art-History as an Academic Study," 1-21; "Sensibility," 22-36; "Vitality," 37-48; "Indian Art," 150-69 tirées des Last Lectures [1939] (Boston, 1962). Voir aussi "Pure and Impure Art" (1924), in Modern Book of Esthetics, M. Roder, ed. (New York, 1964), 304-9 "The Literature of Art," The Burlington Magazine 64, 374 (mai 1934), 242-5; ainsi que la plupart des textes reproduits dans l'ouvrage Post-Impressionists in England sous la direction de Bullen: "Letter," The Burlington Magazine, 12 (mars 1908), 374-6; "Modern French Pictures at Brighton," The Times (11 juillet 1910), 12; "The Grafton Gallery - 1," The Nation (19 novembre 1910), 331; "The Post-Impressionists - 2", The Nation (3 décembre 1910), 402-3; "A Postscript on PostImpressionism", The Nation (24 décembre 1910), 536-7; "Post Impressionism," The Fortnightly Review (mai 1911), 856-67; "The Salons and van Dongen," The Nation (24 juin 1911), 463-4; "Art: The Futurists," The Nation (9 mars 1912), 945-6; "The International Society at the Grafton Gallery," The Nation (20 avril 1912), 87-8; "Introduction, "Catalogue of the Second Post-Impressionist Exhibition (1912), 7-8; "The French Group," Catalogue of the Second Post-Impressionist Exhibition (1912), 13-17; "Art: The Grafton Gallery: an Apologia," The Nation (9 novembre 1912), 249-51; "The Allied Artists," The Nation (2 août 1913), 676-7; "A New Theory of Art," The Nation (7 mars 1914), 937-8.

De Clive Bell, Art [1914] (Toronto, 1987); Since Cézanne [1922] (New York, 1969); Enjoying Pictures - Meditations in the National Gallery and Elsewhere (Londres, 1934); de même que la plupart des textes de Bell reproduits dans Post-Impressionists in England (Bullen): "The English Group", Catalogue of the Second Post-Impressionist Exhibition, 1912, 9-12; "Post-Impressionism \& Aesthetics," The Burlington Magazine, 22 (janvier 1913), 226 230. "The Place of Art in Art Criticism," The Athenaeum, 4714 (3 septembre 1920), 311 -12."The Sculptor's Problem," The Nation \& The Athenaeum, 33, 17 (28 juillet 1923), 543-5; "The Mystery of Manet," The Nation of The Athenaeum, 34, 16 (19) janvier 1924, 570-1. "Gauguin", The Nation of The Athenaeum, 35, 15 (12 juillet 1924), 475-6. "Landmarks in Modern Art III. - Géricault," The Nation of The Athenaeum, 36, 21 (21 février 1925), 713-4."The Colonel's Theory", The Nation \& The Athenaeum, 36, 23 (7 mars, 1925), 779. "Landmarks in Modern Art- IV. - Delacroix," The Nation \& The Athenaeum, 36, 26 (28 mars 1925), 885-6. "The Two Impressionisms," The Nation of The Athenaeum, 40, 14 (8 janvier 1927), 505-7. "Roger Fry's Last Lectures", The New Stateman and The Nation, 18, 452 (21 octobre 1939), 564 .
5 Cézanne, Gauguin, van Gogh pour la première exposition, puis, Matisse et Picasso pour la deuxième. (Voir Jacqueline V. Falkenheim, Roger Fry and the Beginnings of Formalist Art Criticism [1973] (Ann Arbor, 1980), 17, 24.

6 Dans le sens de la définition proposée par Paul Fraisse dans son article "Émotion" de l'Encyclopedia Universalis (France, 1989), VI, 227-230.

7 Lorsque la traduction risquait d'introduire une ambiguïté ou un sens différent de celui généralement porté par l'expression ou par le mot anglais, nous avons utilisé la version originale à l'intérieur de notre texte français. Même si quelques courtes citations sont traduites et intégrées au texte, la plupart des extraits sont cités dans la langue originale. Une traduction libre de l'auteure apparaît en note.

8 Voir à ce propos l'article de Frances Spalding intitulé "Roger Fry and his Critics in a Post-Modernist Age," The Burlington Magazin>, 128, 1000 (juillet 1986), 489-92.

9 "For some years, dit-il, Mr. Fry and I have been arguing, more or less amicably, about the principles of aesthetics. We still disagree profoundly". Bell, Art, xi.

10 Michael Podro, The Critical Historians of Art [1982](New Haven, 1986), xxii.

11 La position de Fry en ce qui concerne les applications possibles des théories de Freud à l'étude des arts reste mitigée. Généralement sceptique, parfois même hostile, Fry prévoit néanmoins une ouverture sur une pertinence qui resterait encore à établir. Historiquement, cette attitude place Fry à mi-chemin entre la méfiance et l'incompréhension générales - constatées par James Strachey dans "The Work of Freud," The New Statesman and Nation, 18, 449 (30 septembre 1939) - et l'enthousiasme de ce même James Strachey (premier traducteur anglais de Freud et proche du Bloomsbury Group).

12 Fry, "An Essay in Aesthetics"(1920) in Vision \& Design [1920] (New York, 1981), 24.

13 Fry, "Essay" in Vision \& Design, 204.

14 Nous conservons la traduction littérale de ce que Fry nomme "imaginative life".

15 Fry, "Essay" in Vision \& Design, 13.

16 Fry, "Essay" in Vision \& Design, 13.

17 Fry, "Essay" in Vision \& Design, 13-14, 19, 23-26.

18 "This imaginative life, which is separated from actual life by the absence of responsive action." Fry, "Essay" in Vision \& Design, 15.

19 "A number of quite interesting but irrelevant things, which in real life could not struggle into our consciousness, bent, as it would be, entirely upon the problem of our apropriate reaction". "Essay" in Vision \& Design, 13-14.

20 Fry, "Essay" in Vision \& Design, 15.

21 "Art is purely a family matter among human beings." Fry, "ArtHistory as an Academic Study" in Last Lectures [1939] (Boston, 1962), 11.

22 Fry, "Art-History" in Last Lectures, 14. 
23 Fry, "Vitality" in Last Lectures, 37-38.

24 Fry, "Essay" in Vision \& Design, 21, 23-25, 26.

25 Bell, $A r t$, xi.

26 Bell, Art, 25.

27 Bell, Art, 278.

28 Bell, Art, 37.

29 "What we feel has nothing to do with a pre-existent mood; we are transported into a world washed clean of all past experience aesthetic or sentimental." Bell, Since Cézanne, 163.

30 "Impersonal and disinterested admiration". Bell, Since Cézanne, 167.

31 Bell, Enjoying Pictures - Meditations in the National Gallery and Elsewhere (London, 1934), 93.

32 Bell, Enjoying Pictures, 97.

33 Pour la métaphysique, voir Art, 49-71, etc. Pour le religieux, voir Art, 75-94, 245, 277, 279, 281, 288, 291 et Enjoying Pictures, 106, etc. Pour l'absolu, voir Art, 90, 266, 291, etc.; Since Cézanne, 162-171, 210, etc. et Enjoying Pictures, 43, 98, 99, 106, etc. Pour la transcendance, voir Art, 82, 277 et Enjoying Pictures, 69.

34 "It appears that the imagination is ready to construct for itself the idea of space in a picture from indications even more vividly than it accepts the idea when given by means of sensual illusion." Fry, "Post-Impressionism", Fortnightly Review, 95 (mai 1911), repris in Post-Impressionists (Bullen), 856-867.

35 "Visual language of the imagination" et "whole lost language of form and colour" dans "Post-Impressionism", repris in PostImpressionists (Bullen), 168 et 178 .

36 Fry, "Post-Impressionism", repris dans Post-Impressionists (Bullen), 173.

37 Les passages sont nombreux, par exemple: "Since the imagination is always most satisfied when it is forced into activity by having to complete the suggestions given to it", puis, "where in both a certain freedom is left to the imagination, where we are moved rather by suggestion than statement", repris de "Some Questions in Esthetics" in Transformations (New York, 1956), 21, 31; ou encore: "where there is nothing but white paper, we carry these movements on the imagination and fill in for ourselves the almost imperceptible indications of the drawing"; à propos des dessins de Bonnard: "He feels the form in its large, easy aspects, and refuses always to define it too clearly, leaving here, too, a certain play to the imagination", tiré de "On some Modern Drawings" in Transformations, 279, 280; ou alors à propos de Matisse: "Our sense of plasticity is no doubt richly appealed to but it is as it were by implication; it demands the active cooperation of the spectator's imaginative power" in Henri-Matisse (Londres, 1930), 15; à propos de Seurat notamment, il reproche les occasions où "The design is affirmed with an almost oppressive decision. We are forbidden to imagine the slightest tremor of change in these impeccable contours", repris de "Seurat" in Transformations, 287.

38 "Now the imaginative life has its own history both in the race and in the individual. In the individual life one of the first effects of freeing experience from the necessities of appropriate responsive action is to indulge recklessly the emotion of self-aggrandisement". Fry, "Essay" in Vision \& Design, 17.

39 Fry associe l'impertinence à un comportement infantile qui doit ĉtre contrôlé et éduqué. Voir, par exemple, "Essay" in Vision \& Design, 17.

40 Fry, "Essay" in Vision \& Design, 17.

41 Fry, "Essay" in Vision \& Design, 17.

42 Fry, Henri-Matisse, 12.

43 Fry, Cézanne, 3-7 et 79-81. Il écrit à la page 11: " $A$ most important characteristic of Cézanne's genius, namely the struggle within him between the Baroque contortions and involutions with which his inner visions presented themselves to his mind, and the extreme simplicity, the Primitive or almost Byzantine interpretation which he gave naturally to the scenes of actual life".

44 Fry, "Some Questions" in Transformations, 57; "Art-History" in Last Lectures, 12; "The Artist's Vision" in Vision \& Design, 33 38. Etc.

45 Fry, "Some Questions" in Transformations, 47-48; Cézanne, 19. 21. Etc.

46 "[Courbet's] innate plastic instinct" expression tirée de "Some Questions" in Transformations, 48; "[Duccio's] instinctive feeling to give such differences of weight and density to his various local colours", un passage de "Plastic Color" in Transformations, 283 et Cézanne's chromatic sensibility in Cézanne, 25.

47 Fry, "Art-History" in Last Lectures, 11, 21.

48 Fry, Cézanne, 33.

49 Méthode utilisée de manière générale dans les Last Lectures, mais présentée de manière plus spécifique à la page 21 .

50 Fry, Cézanne, 83.

51 Fry, "Essay" in Vision \& Design, 17.

52 Fry, "Essay" in Vision \& Design, 17.

53 Fry, "The Literature of Art" (rcvue de Art Now de Herbert Read), The Burlington Magazine, 64, 374 (mai 1934), 245.

54 Fry, "The Literature of Art", The Burlington Magazine, 64, 374 (mai 1934), 245.

55 "Very deep springs of our nature", "deepest aversions", "Essay" in Vision \& Design, 19, 17; "very deep, very vague and immensely generalized reminiscences", "Pure and Impure Art" in Modern Book, 309; "profounder truths" in Cézanne, 28.

56 Sigmund Freud, "The Unconscious" dans The Standard Edition of the Complete Psychological Works of Sigmund Freud (Londres, 1964), 14(1914-1916), 166-215; "Le moi et le ça" dans Oeuvres complètes - Psychanalyse (Paris, 1991), 16 (1921-23), 266.

57 " [...] the Psycho-analysts, with whom I suspect aesthetic enquirers will have increasingly to deal - the Psycho-analysts have given us a very strange and disquieting picture of the contents of this long unnoticed companion of our conscious life. Now they are mainly concerned with mapping out the most primitive and fundamental part of this entity, with those emotional patterns which are laid down in the first years of infancy. These may possibly one day be shown to have a bearing 
upon the nature of artistic creation, but we are more particularly concerned with another aspect, with those parts of the subconscious being which have filtered down through our conscious life and consist of the abiding residue of innumerable sensations, feelings, predilections, aspirations, desires, judgments, in fact all those things which constitute our spiritual life". Fry, "Art-History" in Last Lectures, 14.

58 Fry, "Art-History" in Last Lectures, 14.

59 "Reveal to us first, something about the artist's nervous control, and secondly something of his habitual nervous condition, and finally, something about his state of mind at the moment the gesture was made". Fry, "Sensibility" in Last Lectures, 22-23; voir aussi: "On Some Modern Drawings" in Transformations, 266-281.

60 Fry, "Modern Drawings" in Transformations, 266, 268, ou encore, plusieurs prescriptions quant à la nécessité de contrôler la sensibilité>, dans Last Lectures.

61 Fry établit une distinction entre "sensitivity" et "sensibility". "Sensitivity" fait référence à des qualités spécifiques, reliées à l'exécution de l'oeuvre comme la qualité de la ligne, les relations entre les cons et les couleurs, la facture, la couche, etc.), alors que la"sensibility" implique davantage, plus particulièrement le sentiment (feeling) et les principes qui guident l'organisation et "sous-tendent les variations". Voir "Sensibility" in Last Lectures, 24. Cette distinction reste toutefois floue et les deux aspects se retrouvent souvent sous le mot "sensibility".

62 "These men do succeed in actually saying something". Fry, "Post Impressionism, "in Post-Impressionists (Bullen), 173.

63 Bell, Art, 33-4; Since Cézanne, 154; Enjoying Pictures, 93.

64 "Of any hundred thousand people ninety-nine thousand nine hundred and ninety-nine are born without the aesthetic sense". "The Colonel's Theory", The Nation \& The Athenaeum, 36, 23 (7 mars 1925) 779.

65 L'influence de la philosophie de G.E. Moore sur Clive Bell a été soulignée à plusieurs reprises. L'analyse de George T. Dickie, dans I' article "Clive Bell and the Method of Principia Ethica", The British Journal of Aesthetics, 5, 2 (avril 1965), 139-143), nous apparaît particulièrement jusce.

66 Bcll, Art, 107.

67 Bell, Art, 7, 8, 13, etc.

68 Bell, Enjoying Pictures, 29-30.

69 Bell, Since Cézanne, 158.

70 Fry, "Retrospect" in Vision \& Design, 211.

71 Voir, par exemple, les définitions et variantes présentées dans Lalande, Vocabulaire technique, 278-280.

72 A propos de l'Anthropologie de Kant, voir B. Rimé et K. Scherer, Les Emotions (Paris, 1989), 7.

73 "Great design depends upon emotion, and that, too, of a positive kind, which is nearer to love than hate". Fry, "Art: The Futurists," The Nation (9 mars 1912), in Post-Impressionists (Bullen), 301.

74 "Very stange and disquieting". Fry, "Art-History" in Last Lectures, 14.

75 Bell, Art, 54, 66, 103

76 Bell, Art, 36, 41, 83, 239, 292; Since Cézanne, 95; Enjoying Pictures, 43.
77 Bell, Art, 239; Enjoying Pictures, 43; Art, 82, 158, 277.

78 "A peculiar emotion [...] a particular kind of emotion provoked by works of visual art". Bell, Art, 6, 7 .

79 "I do not mean, of course, that all works provoke the same emotion. On the contrary, every work produces a different emotion. But all these emotions are recognisably the same in kind". Bell, Art, 6-7.

80 Bell, Enjoying Pictures, 9, 15-19, 22, 29-32, etc.

81 Bell, Art, 89, 90.

82 Bell, Art, 3, 20, 25, 30, 33, 36, 37, 51, 68, 148, 158, 240, 284, 288, 292; Since Cézanne, 52, 154, 156, 167, 170; Enjoying Pictures, 10, 11, 15, 49, 68.

83 Bell, Art, 41; Enjoying Pictures, 11, 17, 19; Art, 53; Enjoying Pictures, 11, 16; Since Cézanne, 162; Art, 25, 29, 40; Since Cézanne, 53, 163, 167; Enjoying Pictures 16, 93, 95.

84 Cité par Lalande, Vocabulaire technique, 278, 279.

85 "An emotional confidence, [an] assurance of absolute good, which makes of life a momentous and harmonious whole". Bell, Art, 292.

86 Lalande, Vocabulaire technique, 279-280.

87 Fry, "A New Theory of Art," The Nation (7 mars 1914), in PostImpressionists (Bullen), 489-90.

88 Fry, "Retrospect" in Vision \& Design, 199-200, 206.

89 D'abord, dans "An Essay in Aestherics" (1909), Fry utilise l'expression "aesthetic feeling" et non la fameuse "aesthetic emotion" qui apparaît dans son discours après Art, mais le plus souvent en référence à Art et à la théorie de Bell. Les expressions "aesthetic emotion" ou "esthetic emotion" disparaissent du discours de Fry tout de suite après "Some Questions in Esthetics" dans Transformations (1926) et sont remplacées par des expressions telles que: "plastic feeling" dans Cézanne et dans "Some Questions" in Transformations ou "feeling about form" dans Henri-Matisse ou "specific feeling for plasticity" dans "Art-History" in Last Lectures.

90 Fry, "Essay" in Vision \& Design, 12 -27.

91 Fry, "Retrospect" in Vision of Design, 211; Cézanne, 56 et aussi "Seurat" dans Transformations et dans Last Lectures.

92 Fry, "Retrospect" in Vision \& Design, 211.

93 Fry, "Retrospect" in Vision \& Design, 201.

94 Fry, "Sensibility" in Last Lectures, 36; mais aussi: "Some Questions" in Transformations, 5, 250; Cézanne, 66.

95 Bell, Art, 292.

96 "The perception ofpurposeful order and variety", Fry, "An Essay" in Vision \& Design, 21.

97 "This recognition of purpose is [...] an essential part of the aesthetic judgment proper". Fry, "An Essay" in Vision \& Design, 21.

98 Fry, "Sensibility" in Last Lectures, 29.

99 Fry, "Pure and Impure" (1924), in Modern Book, 308-9.

100 Fry, "Sensibility" in Last Lectures, 28.

101 "It is in this region, then, which lies between rigid order and chaos, that the artist's sensibility functions". Fry, "Sensibility" in Last Lectures, 33.

102 Fry, "A New Theory of Art" in Post-Impressionists (Bullen), 48990. 
103 "Isolate the purely aesthetic feeling from the whole complex of feelings which may and generally do accompany the aesthetic feeling when we regard a work of art". Fry, "Retrospect" in Vision \& Design, 207.

104 Fry,"Retrospect" in Vision \& Design, 210.

105 I had not pushed the analysis of works of art far enough, had not disentangled the purely aesthetic elements from certain accompanying accessories". Fry, "Retrospect" in Vision \& Design, 206.

106 "And what is the value of this elusive and - taking the whole mass of mankind - rather uncommon aesthetic emotion [...] ?Fry, "Retrospect" in Vision \& Design, 211.

107 Fry, "Retrospect" in Vision \& Design, 211.

108 "We have no need for our purposes to create the hypothesis of any mysterious or specific faculty". Fry, "Some Questions" in Transformations, 7.

109 Fry, "Some Questions" in Transformations, 3, 4 , 7.

110 Selon la formulation de 1909, dans "An Essay in Aesthetics".

111 Cité par Lalande, Vocabulaire technique, 278-280.

112 Piaget, "Les relations entre l'intelligence et l'affectivité dans le développement de l'enfant", dans Rimé et Scherer, Les émotions, 78 .

113 Piaget, "Les relations", dans Rimé et Scherer, Les émotions, 92.

114 "Art and Religion are, then, two roads by which men escape from circumstance to ecstasy", ou, "It is not as sanctuaries from life sanctuaries devoted to the cult of aesthetic emotion - [...] that museums and galleries become noxious". Bell, Art, 267.

115 "Because the aesthetic emotions are outside and above life, it is possible to take refuge in them from life". Bell, Art, 292.

116 "Those who take art seriously are those who find in art an escape from life". Bell, Enjoying Pictures, 106.

117 Bell, Art, 11.

118 Bell, Enjoying Pictures, 85-6.

119 "Those moments [when] I lose myself in that infinitely sublime state of mind to which pure visual form transports me". Bell, Art, 31 .

120 "He who has once lost himself in an 'O Altitudo' [...] He who can withdraw into the world of ecstasy will know what to think of circumstance". Bell, Art, 292.

121 "That supreme rapture in which we are carried to a world of impersonal and disinterested admiration". Bell, Since Cézanne, 167.

122 Those who take art seriously [...] are carried out of themselves, out of this world, into the world of the spirit". Bell, Enjoying Pictures, 106.

123 Bell, Art, 91.

124 Arthur Schopenhauer, "Le monde comme volonté et comme représentation", tiré du Vouloir-vivre, l'art et la sagesse, textes choisis par André Dez (Paris, 1989), 97.

125 Schopenhauer, Le vouloir-vivre, 98.
126 Schopenhauer, Le vouloir-vivre, 157.

127 Schopenhauer, Le vouloir-vivre, 215 et 218.

$128 \mathrm{~J}$. Laplanche et J.-B. Pontalis, Vocabulaire de la psychanalyse (Paris, 1967), 122-123 et 150-151.

129 Laplanche \& Pontalis, Vocabulaire de la psychanalyse, 332-335 et 341-343.

130 Laplanche \& Pontalis, Vocabulaire de la psychanalyse, 241-254, 341-342.

131 Schopenhauer, Le vouloir-vivre, 218.

132 Fry, "Essay" in Vision \& Design, 19-20.

133 Fry, "Essay" in Vision \& Design, 20.

134 Fry, "Retrospect" in Vision \& Design, 204-5.

135 Fry, "Art-History" in Last Lectures, 11, 13 et 15.

136 Fry, "Essay" in Vision \& Design, 21.

137 "The ideal transaction would be one in which the artist embodied his 'experience' completely in the work of art and met with a spectator capable of perfect response to that experience". Fry, "Art-History" in Last Lectures, 15.

138 Fry, "Art-History" in Last Lectures, 15.

139 "If the artist is one with whom we can communicate. Fry, "Sensibility" in Last Lectures, 29.

140 "The only means by which human beings can communicate to each other the quality and quiddity of their experiences". Fry, "ArtHistory" in Last Lectures, 11.

141 Laplanche \& Pontalis, Vocabulaire de la psychanalyse, 336-9, 341 343.

142 "[...] The value of my method is to stop that immediate like or dislike response to the work of art as a whole which as we have seen is just as likely to be due to imperfections in our receiving set as to anything in the work of art itself. It is possible, I think, by some such methods to circumvent our native prejudices and predilections and to acquire a more alert passivity in our attitude". Fry, "Art-History" in Last Lectures, 21.

143 "[...] in a great work of art we get a conviction that we could go on with our endless sequence of such questionings going down to the minutest particulars of the picture and for ever having them resolved". Fry, "Sensibility" in Last Lectures, 30.

144 Laplanche \& Pontalis, Vocabulaire de la psychanalyse, 256-9.

145 Schopenhauer, Le vouloir-vivre, 105.

146 Schopenhauer, Le vouloir-vivre, 106.

147 Schopenhauer, Le vouloir-vivre, 105.

148 Fry, "Pure and Impure" in Modern Book, 309.

149 Schopenhauer, Le vouloir-vivre, 218.

150 Louis Marin, "L'oeuvre d'art et les sciences sociales" dans "Les Enjeux" (vol. IV), Encyclopaedia Universalis, (Paris, 1990), $947-$ 969.

151 Marin, "L'oeuvre d'art et les sciences sociales", 969. 